\title{
Impact of Probiotic B. Infantis EVC001 Feeding in Premature Infants on the Gut Microbiome, Nosocomially Acquired Antibiotic Resistance, and Enteric Inflammation
}

\section{Marielle Nguyen}

Kaiser Permanente

Heaven Holdbrooks

Kaiser Permanente Southern California

Prasanthi Mishra

Kaiser Permanente Southern California

Maria Abrantes

Kaiser Permanente Southern California

Sherri Eskew

Kaiser Permanente Southern California

MariaJamiela Garma

Kaiser Permanente Southern California

Cyr-Geraurd Oca

Kaiser Permanente Southern California

Carrie McGuckin

Evolve Biosystems

Cynthia Hein

Evolve Biosystems

Ryan Mitchell

Evolve Biosystems

\section{Sufyan Kazi}

Evolve Biosystems

\section{Stephanie Chew}

Evolve Biosystems

Giorgio Casaburi

Evolve Biosystems

Heather Brown

Evolve Biosystems

\section{Steve Frese}

University of Nebraska-Lincoln 
Bethany Michele Henrick ( $\square$ bhenrick2@unl.edu )

Evolve Biosystems, Inc. https://orcid.org/0000-0003-1883-6749

\section{Research}

Keywords: Preterm infant, gut microbiome, Bifidobacterium longum subspecies infantis EVC001, antibiotic resistant genes (ARG), enteric inflammation

Posted Date: October 20th, 2020

DOI: https://doi.org/10.21203/rs.3.rs-92208/v1

License: (c) (1) This work is licensed under a Creative Commons Attribution 4.0 International License. Read Full License 
1 Impact of probiotic $B$. infantis EVC001 feeding in premature infants on the gut microbiome, nosocomially

2 acquired antibiotic resistance, and enteric inflammation

4 Nguyen, Marielle ${ }^{1,2}$; Holdbrooks, Heaven ${ }^{1}$; Mishra, Prasanthi ${ }^{1,2}$; Abrantes, Maria A. ${ }^{1,2}$; Eskew, Sherri ${ }^{1}$;

5 Garma, Mariajamiela ${ }^{1}$; Oca, Cyr-Geraurd ${ }^{1}$; McGuckin, Carrie ${ }^{3}$; Hein, Cynthia B. ${ }^{3}$; Mitchell, Ryan D. ${ }^{3}$;

6 Kazi, Sufyan ${ }^{3}$; Chew, Stephanie ${ }^{3}$; Casaburi, Giorgio ${ }^{3}$; Brown, Heather K. ${ }^{3}$; Frese, Steven A. ${ }^{3,4, *}$, and

7 Henrick, Bethany M. ${ }^{3,4, *}$

8

$10 *$ Co-corresponding Authors:

11 Bethany M. Henrick, $\mathrm{PhD}$

12 Director, Immunology \& Diagnostics, Evolve Biosystems, Inc.

13 Adjunct Assistant Professor

14 Department of Food Science and Technology, University of Nebraska Lincoln

15 916-479-0226

16 bhenrick2@unl.edu

17

18 Steven A. Frese, $\mathrm{PhD}$

19 Director, Microbiology \& Bioinformatics, Evolve Biosystems, Inc.

20 Adjunct Assistant Professor

21 Department of Food Science and Technology, University of Nebraska Lincoln

22 717-418-3254

23 steven.frese@,unl.edu

24

25

26

27

28

29

30

31

32

33

34

35

36

37

38

39 
Abstract

Background: Preterm birth is a major determinant of neonatal survival and morbidity, but the gut microbiome and associated enteric inflammation are also key factors in neonatal development and the risk

44 of associated morbidities. We prospectively and longitudinally followed two cohorts of preterm infants, 45 one of which was fed Bifidobacterium longum subsp. infantis (B. infantis) EVC001 daily, and the other was 46 not fed a probiotic. Hospital feeding protocol assigned all infants born at less than $1500 \mathrm{~g}$ and/or 34 weeks

47 corrected gestational age to the probiotic feeding protocol, whereas infants born at $>1500 \mathrm{~g}$ and/or 34 weeks 48 corrected gestational age were not fed a probiotic. Fecal samples collected opportunistically 49 (approximately 2 samples per week) throughout the hospital stay were analyzed from 292 samples collected 50 from 77 infants. Fecal samples were subjected to shotgun metagenomic sequencing and quantification of 51 enteric inflammation markers. We also collected de-identified metadata from patient medical records.

52 Results: The gut microbiome of preterm infants was typified by a high abundance of Enterobacteriaceae and/or Staphylococcaceae and multivariate modeling identified the probiotic intervention, rather than degree of prematurity, day of life, or other clinical interventions as the primary source of change in the gut microbiome. Among infants fed B. infantis EVC001, a high abundance of total Bifidobacteriaceae developed rapidly, the majority of which was B. infantis confirmed via subspecies-specific qPCR.

57 Associated with this higher abundance of Bifidobacteriaceae, we found increased functional capacity for 58 utilization of human milk oligosaccharides (HMOs), as well as reduced abundance of antibiotic resistance 59 genes (ARGs) and the taxa that harbored them. Importantly, we found that infants fed B. infantis EVC001 60 experienced diminished enteric inflammation, even when other clinical variables were accounted for using 61 multivariate modeling.

62 Conclusion: These results provide an important observational background for probiotic use in a NICU 63 setting, and describe the clinical, physiological, and microbiome-associated improvements in preterm 64 infants associated with $B$. infantis EVC001 feeding.

65 Keywords: Preterm infant, gut microbiome, Bifidobacterium longum subspecies infantis EVC001, 66 antibiotic resistant genes (ARG), enteric inflammation 
Preterm birth, defined as less than 37 weeks' gestation age, accounts for 1 in 9 live births in the

69 United States and is associated with an immature gastrointestinal tract, diminished barrier function, and 70 underdeveloped immune function leading to increased morbidity and mortality compared to term infants.

71 Unlike infants born full-term, premature infants have extended stays in the hospital environment of the

72 Neonatal Intensive Care Unit (NICU), increased exposure to associated clinical protocols including 73 antibiotics, proton pump inhibitors, parenteral nutrition, and limited access to human milk all of which 74 dramatically shapes gut microbiome composition [1,2]. Importantly, recent work has shown that preterm 75 infants are rapidly colonized by nosocomial, antibiotic-resistant bacteria associated with an increased risk 76 of serious infection and death [3]. These bacteria confer negative impacts on neonatal growth and 77 development [1] and are associated with an increased risk of necrotizing enterocolitis (NEC) [4,5] and late78 onset sepsis [6-8]. Importantly, increased prevalence of Proteobacteria has been shown to precede NEC $79[4,8,9]$ and exacerbate enteric inflammation [10]. Proteobacteria, broadly, have also been associated with 80 the pathogenesis of sepsis and NEC in premature infants [11-13]. Although the exact mechanisms by which 81 these bacteria evoke heightened inflammatory responses in the preterm infant gut are not completely 82 understood, sub-optimal mucosal integrity and/or predisposition toward an exaggerated inflammatory 83 profile, including exacerbated IL-8 cytokine production, has been observed in preterm infants [14]. Indeed, 84 exaggerated inflammatory responses driven by nosocomially-derived microbes including Proteobacteria 85 has led to a desire to find strategies, including the use of probiotics, to mitigate the risks associated with 86 prematurity that have etiological links to the gut microbiome.

87 A recent observational study comparing longitudinal fecal samples taken from preterm infants 88 showed that supplementation with Bifidobacterium and Lactobacillus remodeled the gut microbiome, 89 replicating a gut microbiome more closely resembling that of a term infant. However, physiological effects 90 on the host were not determined [15], and distinct species-specific effects among Bifidobacterium are 91 beginning to emerge that distinguish the ability among Bifidobacterium species to modulate host enteric 92 inflammation and epithelial integrity from those that do not $[16,17]$. Beyond observational studies, 
93 extensive evidence has indicated a beneficial role of feeding Bifidobacterium longum subsp. infantis (B.

94 infantis) to premature infants, with significant decreases in morbidity and mortality reported, including

95 those from NEC [18-20]. In animal models, B. infantis supplementation was effective at reducing NEC

96 injury scores and minimizing intestinal inflammation [21,22]. Furthermore, establishing B. infantis

97 abundance in the infant gut restores important ecosystem services of the microbiome that are beneficial to

98 infant health [23]. Particularly, the efficient fermentation of human milk oligosaccharides (HMOs) into

99 host-accessible organic acids (e.g. acetate and lactate), helps reduce degradation of gastrointestinal mucin,

100 and results in significant reductions of bacterial populations with pathogenic potential [16,23-26].

101 Moreover, when B. infantis is compared directly to other probiotic bacteria, including Bifidobacterium

102 animalis subsp. lactis (B. lactis), it showed superior ability to colonize the premature infant gut [27], based

103 on its ability to utilize the full suite of selective prebiotic carbohydrates found in human milk (HMOs).

104 Therefore, early establishment of a gut microbiome that limits growth of bacteria associated with

105 detrimental outcomes, maximizes nutrition, and reduces inflammation during a key developmental window

106 is particularly important to preterm neonatal health.

107 To examine the effect of feeding B. infantis EVC001 to a preterm infant population, we 108 prospectively and longitudinally collected fecal samples from 77 infants born before 37 weeks 109 postmenstrual age and compared the gut microbial composition and development, as well as enteric 110 inflammation profiles. These infants were assigned to one of two distinct, hospital-directed feeding 111 protocols based on gestational age at birth and weight at birth. One feeding protocol dictating daily feedings 112 of $B$. infantis EVC001 (EVC001-fed; $<32$ weeks corrected gestational age and/or $<1500$ grams; $\mathrm{n}=31$ ) and 113 a second feeding protocol was used for premature infants born after 32 weeks corrected gestational age and $114>1500 \mathrm{~g}$ at birth ("No probiotic"; n=46; Fig. 1a). All infants were predominantly fed human milk but were 115 discordant for probiotic use based on their corrected gestational age. Further, we collected deidentified 116 patient metadata to compare the impact of other clinical interventions on the preterm infant gut microbiome 117 and to account for known differences between the feeding cohorts. Here, we find that EVC001-fed 118 premature infants had increased colonization of Bifidobacterium in their gut microbiome, an increase in 
119 genes conferring efficient utilization of HMOs, significantly decreased overall abundance of antibiotic-

120 resistant (bacterial) genomes (ARGs), and importantly, a decreased enteric inflammatory profile compared

121 to premature infants not fed a probiotic, after accounting for other confounding clinical variables.

\section{Results}

\section{Demographics and clinical observations of the preterm infant subjects}

Seventy-seven (77) preterm infants were enrolled from May to October 2019 from Kaiser

126 Permanente Orange County Anaheim Medical Center (Anaheim, CA USA; Level 3 NICU, 25 beds) and

127 Kaiser Permanente Orange County Irvine Medical Center (Irvine, CA USA; Level 2 NICU, 10 beds). No

128 changes to the hospital standard of care were introduced by participation in this prospective sampling study.

129 Participant demographic and clinical data are provided in Table 1. Per hospital feeding protocols, infants

130 born at less than 32 weeks gestational age (GA) or at less than 1500 grams birth weight received $B$. infantis

131 EVC001 with MCT oil, via orogastric or nasogastric tube starting at the initiation of trophic feeds and

132 continued daily through 34 weeks corrected gestational age (cGA, n=31). Infants born after 32 weeks GA

133 did not receive $B$. infantis EVC001 or any other probiotic (n=46; Fig. 1a). Thus, the two study groups

134 differed in general clinical acuity, both in feeding protocols and the level of acuity between hospitals. Given

135 the differences in gestational age $(P<0.001)$, there was also a corresponding difference in birthweight $(P$

$136<0.001$ ) but both groups received a predominantly human milk diet via maternal, donor and/or commercial

137 human milk through 34 weeks cGA and the infants born at an older GA not fed probiotics received more

138 infant formula as a result $(P<0.001)$. Also, significantly more fecal samples were collected from the

139 infants fed $B$. infantis EVC001 compared to infants not fed probiotics (6.23 vs 2.26 on average, $P<0.001)$

140 due to the generally longer duration of stay of the infants born more premature, and infants born at an earlier

141 gestational age received more inhaled steroids, owing to their prematurity (e.g. albuterol, budesonide,

142 levalbuterol, ipratropium; $22 \%$ vs. $0.9 \%, P<0.001$ ). Feeding $B$. infantis EVC001 was well tolerated and

143 no adverse events related to probiotic consumption were observed. 


\section{Alteration of microbiome composition in preterm infants fed B. infantis EVC001}

Shotgun metagenomic sequencing was performed on 292 samples, yielding an average cluster of 33,658,645 ( \pm SD 7,269,047.16) and totaling 67,317,290 ( \pm SD 14,538,094.3) reads per sample. After quality-filtering and removal of reads mapping to the human genome using GenCOF [28], 33.6 million

149 sequences per sample $( \pm 7.8$ million, SD) were processed for microbial taxonomy and functional 150 classification. Using a cross-validated clustering approach, three broad compositional cluster types were 151 identified (Fig. 1b; see Methods). Typical communities in these cluster types were predominantly composed 152 of either Bifidobacterium longum species (which includes B. infantis; 56.91\%, +/- 20.97\% SD), Escherichia 153 coli $(56.14 \%,+/-16.00 \%$ SD) and unclassified Escherichia $(19.11 \%,+/-6.88 \%)$, or a more variable mix 154 of taxa that included Staphylococcus epidermidis (14.22\%, +/- 27.51\% SD), Klebsiella pneumoniae 155 (23.54\% +/- 37.15\% SD) and Enterococcus faecalis $(10.73 \%,+/-24.86 \%$ SD; Fig. 1b). Congruent with the higher sample frequency from infants fed $B$. infantis EVC001, the majority of the samples fell into the

157 high B. longum species cluster type $(\mathrm{n}=160)$, with other samples split between the Escherichia cluster type $158(n=63)$ and the more variable cluster type characterized by S. epidermidis $(n=69)$. Samples from infants 159 fed B. infantis EVC001 were not evenly distributed among the cluster types, with $90 \%$ of samples from the 160 B. longum species cluster having originated from infants fed B. infantis EVC001, and only $37 \%$ and 33\% 161 of samples from the Escherichia species cluster and mixed S. epidermidis cluster were from infants fed $B$. 162 infantis EVC001, respectively.

163 In order to account for clinical variables associated with prematurity, and which differed between 164 infants assigned to each feeding protocol, we used a boosted, additive linear mixed effects model 165 (MaAsLin2; https://huttenhower.sph.harvard.edu/maaslin/) to independently assess the effect that each of 166 these clinical variables had on the gut microbiome. When accounting for the individual effect of clinical 167 variables (i.e. probiotic feeding, inhaled steroid use, diaper rash, recent antibiotic exposure, diet, TPN 168 intake, age at sampling, weight at birth, gestational age at birth), we found that while several of them (diaper 169 rash, infant formula in diet, human milk in diet, age at sampling) did have small, but significant associations 
170 with various bacterial families, the size of the effect of these clinical variables was quite low when compared

171 to probiotic feeding (Fig. 2a). Notably, the introduction of B. infantis EVC001, independent of other clinical

172 variables, was associated with $27.6 \%$ more Bifidobacteriaceae abundance, when all other clinical variables

173 were controlled (FDR-adjusted $P$ value $=0.000183$; Fig. 2a). Second, while formula feeding (any amount)

174 was associated with less Staphylococcaceae $(-3.65 \%$, FDR-adjusted $P$ value $=0.0112$ ), other associations

175 included age at sampling (day of life), formula feeding, human milk feeding volume ( $\mathrm{mL} / \mathrm{kg}^{*}$ day), and

176 diaper rash, all of which were associated with significant differences in the gut microbiome (FDR-corrected

$177 P<0.05$ ), but these associations accounted for less than a $1 \%$ change per unit increase (e.g. $\mathrm{mL} / \mathrm{kg} * \mathrm{day}$,

178 week, or day, as indicated; Fig. 2a).

As inclusion of feeding $B$. infantis EVC001 into the diet of infants had the largest individual impact

on gut microbiome composition (Fig. 2a), we also sought to understand how Bifidobacteriaceae abundance interacted with other taxonomic groups among infants fed EVC001. Broadly, increased Bifidobacteriaceae abundance was strongly associated with diminished Enterobacteriaceae abundance (which includes the correlations between $B$. longum species and each of two Enterobacteriaceae species among infants fed $B$. infantis EVC001 (respectively, Spearman's $\rho=-0.38, P<0.001$, Spearman's $\rho=-0.26, P<0.001$; Fig. 2c,

188 d). This was further corroborated by comparing with species-specific qPCR for $B$. infantis with the 189 abundance of these bacteria at different taxonomic levels (Supplementary Fig. 1).

191 from the others, we also explored how interactions between clinical variables and the gut microbiome were

192 further interrelated. B. infantis is known to utilize human milk oligosaccharides as part of its colonization 193 of the gut microbiome, so we examined whether a greater abundance Bifidobacteriaceae was associated 194 with increased rates of human milk feeding. There was a significant correlation between the volume of 195 human milk in an infant's diet and the abundance of $B$. longum species (Spearman's $\rho=0.26, P<0.001$ ), 
but only among infants who had been fed $B$. infantis EVC001 (Supplementary Fig. 2a). Infants who were

197 not assigned to the feeding protocol that included B. infantis EVC001 did not have a significant association

198 between human milk feeding volume and the abundance of Bifidobacteriaceae (Supplementary Fig. 2b;

199 Spearman's $\rho=0.046, P=0.649)$. The magnitude and relationship between $B$. longum species and human

200 milk feeding volume only among infants fed EVC001 was confirmed by subspecies-specific qPCR for $B$.

201 infantis (Spearman's $\rho=0.34, P<0.001$; Fig. S2c-d).

\section{Gut microbiome development differs among infants fed EVC001}

After identifying limited impacts of differing clinical variables between the two feeding cohorts apart from probiotic use and the associated increased abundance of Bifidobacteriaceae (i.e. B. infantis; Fig.

2a), which was associated with diminished abundance of other taxa typical for the preterm infant gut microbiome (Fig. 2b-d), we examined the microbiome composition of infants in both feeding cohorts over time. Fecal samples from EVC001-fed infants developed a gut microbiome distinct from preterm infants not fed a probiotic. In addition to distinct functional differences, overall taxonomic composition differed,

210 though a wider range of $B$. infantis relative abundances were observed when compared to previous studies

211 examining the organism in healthy breastfed infants born at term [24]. Taxonomic classification across all 212 samples identified variable community compositions predominantly composed of Escherichia $(32.59 \%(+/-$ $21340.00 \%$ SD) vs $9.95 \%$ (+/- $23.58 \%$ SD)), Klebsiella $(12.09 \%(+/-25.61 \%$ SD) vs. $22.52 \%(+/-28.35$ SD),

214 Bifidobacterium (11.50\% (+/- 24.10\% SD) vs. 47.50\% (+/- 27.59\% SD)), Staphylococcus (11.35\% (+/$21527.07 \%$ SD) vs. $3.24 \%(+/-9.53 \%)$, Enterobacter $(4.64 \%(+/-16.28 \%$ SD) vs. 5.25\% (+/- 15.72\%SD)), 216 and Enterococcus $(7.73 \%(+/-17.76 \%$ SD) vs. 3.29\% (+/- 10.02\% SD) when comparing samples from 217 infants not fed B. infantis EVC001 to infants fed the probiotic. 
Bifidobacteriaceae, despite human milk feeding (Fig. 3a). Notably, infants who were less than $1500 \mathrm{~g}$ at

221 birth were assigned to the feeding protocol which included B. infantis EVC001 and these infants also 222 developed a high abundance of $B$. infantis (Subjects 302 and 335; Fig. 3a). Accordingly, the species cluster 223 identity of samples from infants fed $B$. infantis EVC001 were predominantly composed (76\%) of the $B$.

224 longum species cluster (Fig. 3b) and this increased as infants reached maximum human milk feeding 225 volumes (33.8 weeks median corrected gestational age) to $81 \%$. In contrast, samples from infants not fed 226 B. infantis EVC001 were primarily identified as belonging to the Escherichia species or S. epidermidis 227 species clusters by the time they reached the maximum human milk feeding volumes (38\% and $49 \%$, 228 respectively, at 36.1 weeks median corrected gestational age), and this was consistent with the sample 229 identity overall from infants not fed B. infantis EVC001, representing $40 \%$ and $46 \%$ of samples collected 230 from these infants, respectively (Fig. 3b). We corroborated these findings by examining the absolute 231 abundance of $B$. infantis via species-specific qPCR (See Methods). Similar to results obtained by 232 metagenomic sequencing, we found that among infants fed B. infantis EVC001, B. infantis levels rapidly 233 increased over time (Fig. S3) and reached maximum levels, in terms of both absolute and relative 234 abundance, as infants approached discharge from the hospital (Fig. S3).

\section{B. infantis EVC001 feeding improves functional capacity of preterm infant gut microbiomes for}

\section{HMO utilization}

Given infants in the study population were fed human milk through 34 weeks cGA or longer, with or without human milk-based fortification and/or formula, we conducted an exploratory analysis using the KEGG Orthology (KO) database of functional orthologs to identify 37 representative key functions

241 necessary to metabolize human milk oligosaccharides (e.g. oligosaccharide binding proteins, sialidases, 242 fucosidases, lacto-N biosidase, etc.) identified by Sela et al (2008) [29] (Fig. 4a). We then compared the 243 distribution of these functions across each of the sample clusters and examined the impact of $B$. infantis 
244 EVC001-feeding on the abundance of these functions. A comparative analysis indicated the relative 245 amount (counts per million, CPM) of KO functions related to HMO metabolism were globally enriched 246 among samples from infants that belonged to the B. longum species cluster (Fig. 4b).

In B. infantis, glycolytic enzymes are internally localized and require complex HMO-transport 248 systems (e.g. ABC transporters; green, Fig. 4a) to facilitate efficient uptake of HMO structures into the 249 bacterial cell [30]. Functions in this category were significantly more abundant among samples from 250 EVC001-fed and from samples belonging to the B. longum-species cluster (Fig. 4b, 4c). While some 251 Bifidobacterium species are able to externally degrade carbohydrates and transport the constituent 252 monomers and dimers (e.g. B. bifidum [31]) and others are only able to access HMOs by the glycolytic 253 activity of other bacteria (e.g. B. breve [32]), the mean abundance of these Bifidobacterium among these 254 samples was very low $(1.7 \%$ B. breve, $0.142 \%$ B. dentium, and others below $0.01 \%)$ and rare (only three 255 subjects had $>30 \%$ B. breve at any time, only one had $>30 \%$ B. dentium). These data emphasized both the 256 rarity of Bifidobacterium among NICU patients absent probiotic intervention and the relative importance 257 of the carbohydrate transport systems to enable HMO utilization by Bifidobacterium present in these 258 samples.

Among the functions necessary for HMO utilization by the gut microbiome, glycolytic enzymes 260 including fucosidases and sialidases are indispensable for the primary removal of terminal fucose and sialic 261 acid moieties from complex HMO molecules (blue, Fig. 4a). These functions enable degradation of intact 262 HMO structures into their constituent fragments and the transfer of these fragments to fermentation 263 pathways (Fig. 4a). Glycolytic functions were significantly enriched among infants in the $B$. longum species 264 cluster and among EVC001-fed infants in other cluster types (teal, Fig. 4a) as were key biosynthetic steps 265 in the cleavage of core I and core II HMO glycans, Lacto- $N$-biose and $N$-acetyllactosamine (e.g. K013509) 266 via sugar kinases, as well as utilization of sialic acid (e.g. K01788), and $N$-acetyl-hexoses (e.g. K15533) 267 (Fig. 4c). Together, these results demonstrate greater primary HMO degradation potential among infants 
colonized by B. longum species (Fig. 4c), and particularly, among EVC001-fed infants as all general

269 functions were significantly enriched $(P<0.0001)$ among these samples (Fig. 4d).

B. infantis also has genes enabling the transport of urea and the hydrolysis of urea to ammonia co-

271 localized in the genome with HMO metabolism [29]. As human milk naturally contains urea (45

$272 \mathrm{uMol} / \mathrm{L})[33$, the liberation of ammonia from urea as a nitrogen source has been proposed for $B$. infantis

273 [29]. Genes involved in urea metabolism (urea transport and hydrolysis; orange, Fig. 4a) were significantly

274 enriched among samples from infants belonging to the B. longum species cluster type (Fig. 4b), and among

275 EVC001-fed infants (Fig. 4c). Samples from infants that were classified in the mixed S. epidermidis cluster

276 type were also enriched for urease transport and activity, relative to the Escherichia species cluster type.

277 Among the Gram-positive organisms, which were comparatively enriched among these samples, urease

278 activity is chiefly thought to be a pH-buffering mechanism [34].

Other functions identified in B. infantis HMO-related gene clusters included genes related to

280 regulatory functions (e.g. a lacI homolog, purple, K02529; and a protein involved in lysine metabolism (4-

281 hydroxy-tetrahydrodipicolinate synthase), pink, K01714) were also significantly more abundant $(P<$

282 0.0001) among samples from infants in the B. longum species group and among samples from infants fed

283 B. infantis EVC001 (Fig. 4c, 4d).

\section{Antibiotic resistance genes are acquired during NICU stays and differ between hospital NICUs}

As the spread of antibiotic-resistant bacteria is of particular concern in clinical practice, we examined the abundance of antibiotic resistance genes (ARGs) in samples collected from infants in this

287 study. We first profiled the taxonomic composition of ARGs in all stool samples (i.e., the resistome). Nine 288 species were responsible for carrying $86.2 \%$ of the total ARGs identified in the study, while $4.5 \%$ of ARGs 289 could not be confidently assigned to a single species (Fig. 5a). The nine species belonged to the families of 290 Enterobacteriaceae, Enterococcaceae and Staphylococcaceae. Escherichia coli harbored 48.5\% of the total 291 resistome, followed by Klebsiella pneumonia (9.6\%), Staphylococcus aureus (7.5\%) and Enterobacter 
cloacae (5.7\%). No significant differences in the relative abundance of bacterial species harboring ARGs 293 were found between the hospitals studied here (Fig. 5a).

Next, we investigated unique ARGs in each NICU and found that of the 315 genes identified, 204

295 were common in both units, while 19 ARGs were specific to Irvine Medical Center and 92 were specific to

296 the Anaheim Medical Center (Supplementary Table ARG 1). However, only the abundance of four ARGs

297 was significantly different when accounting for sample distribution between hospitals. Specifically, the

298 abundance of aadA5 (ARO:3002605), an aminoglycoside nucleotidyl transferase known to confer

299 resistance to streptomycin/spectinomycin and encoded by plasmids, transposons and integrons in E. coli,

300 K. pneumoniae, P. aeruginosa and E. cloacae [35], was significantly more abundant in the Irvine compared

301 to the Anaheim NICU ( $P=0.01$, Bonferroni). The aminoglycosides resistance gene, aph(2'')-IIIa

302 (ARO:3002636)[36], was also unique to the Irvine Medical Center. Conversely, the level of gene $A A C(6)$ -

303 Ic (ARO:3002549), known to confer aminoglycosides resistance in Klebsiella ssp [36] was significantly

304 more abundant in the Anaheim compared to Irvine NICU $(P=0.03$; Bonferroni).Finally, ARO: 3003291

305 was the only significantly different ARG found within both hospitals and was 1.2-fold higher in the Irvine

306 NICU $(P=0.02$; Bonferroni). This gene is known as Staphylococcus aureus rpoC conferring resistance to

307 daptomycin, a lipopeptide antibiotic with potent activity against Gram-positive bacteria [37]

308 (Supplementary Table ARG 1).

309 While there was not a difference in the taxonomic identity of ARGs between hospitals, we did

310 identify ARGs specific to each hospital site (Supplementary Table ARG 1). Four B. infantis EVC001-fed

311 infants were transferred from the Anaheim Medical Center to the Irvine Medical Center at different times

312 throughout the duration of the sample collection period, and we found all four infants acquired unique,

313 hospital-specific ARGs after transferring to the new hospital (Fig. 5b; Supplementary Table ARG 2).

314 Importantly, these four infants were colonized with novel hospital-specific ARGs acquiring 66 new ARGs,

315 on average, in 48 days dependent on NICU length of stay. Importantly, these four transferred infants

316 reached ARG levels comparable to infants under the same feeding protocol including B. infantis EVC001 
317 and maintained fewer ARGs than infants on the feeding protocol that did not include B. infantis EVC001 318 (Fig. 5b).

Colonization by B. infantis EVC001 is associated with a reduced antibiotic-resistant gene burden in 321 preterm infants identified against the CARD database was $0.04 \%$ in the group not fed the probiotic and $0.008 \%$ in the

324 EVC001-fed infants, a difference in mean ARG abundance of $80.6 \%(P<0.0001$; Fig. 5d). A total of 315

325 unique ARGs were identified among all samples from the CARD database (Supplementary Table ARG 3).

326 Of those, 85 ARGs were differentially abundant between EVC001-fed infants and those who were not fed

327 probiotics $(P<0.05$; Bonferroni). Eighteen unique ARGs were significantly more abundant in the EVC001-

328 fed (mean 1.21-fold higher) compared to no probiotic preterm infants (Supplementary Table ARG 4).

329 Conversely, 67 unique ARGs were significantly more abundant (270-fold higher, on average) in the no

330 probiotic compared to EVC001-fed preterm infants. In particular, three ARGs were several orders of 331 magnitude lower in abundance among the samples from EVC001-fed infants compared to preterm infants 332 not fed the probiotic. The gene cat (ARO:3002670) was $10^{4}$-fold less abundant $(P=0.005$; Bonferroni) in 333 EVC001-fed compared to infants not fed a probiotic. Cat is a well-known chloramphenicol 334 acetyltransferase gene described in a range of bacteria including Enterococcus and Staphylococcus spp. 335 conferring resistance to phenicol antibiotics [38,39]. Mpha (ARO:3000316) is a gene that encodes for the 336 resistance enzyme MPH (2')-I, known to inactivate multiple macrolides (e.g. azithromycin, erythromycin) 337 in species including Enterobacter cloacae, Escherichia coli, Klebsiella oxytoca and Klebsiella pneumoniae. 338 Mpha was found to be $4.6 \times 10^{3}$ fold lower among EVC001-fed compared to preterm infants not fed the 339 probiotic (Bonferroni corrected $P=0.01$; Bonferroni). Of interest, only 10 preterm infants in the no 340 probiotic group were known to have received erythromycin during their clinical stay. Mrx (ARO:3003839, 341 recently updated as ARO:3000333) was $3 \times 10^{3}$ fold lower in EVC001-fed compared to no probiotic preterm 342 infants $(P=0.007$; Bonferroni). Mrx is also associated with macrolide resistance and is part of the gene 
cluster $m p h A-m r x-m p h R$. The majority $(70 \%)$ of the significant ARGs were classified as multidrug resistant.

344 Forty-eight multidrug-ARGs were found to be on average 277-fold lower in EVC001-fed infants when

345 compared to infants who were not fed the probiotic, which are known to confer resistance to several drug 346 classes (14 different drug classes and up to 29 on average).

347 Finally, given infants in early life acquire microbes from the environment over time (Fig. 5c), we 348 built a mixed effect linear model considering time as a function equal to the preterm infant's day of life 349 when a sample was collected, respectively, to assess the rate of nosocomial ARG acquisition. We also 350 considered whether feeding $B$. infantis EVC001 or the NICU location at the time of collection would have 351 an impact on the model while controlling for individual subject variation. Our model predicted that with all 352 other variables held constant in an approximate linear model, infants acquired on average a new ARG-type 353 for every two days spent in the NICU $(P<0.0001)$. This finding was consistent between hospitals $(P>$ 354 0.05). Among preterm infants fed B. infantis EVC001, there were 13.6 fewer ARG types, on average, 355 compared to preterm infants who were not fed the probiotic throughout their entire NICU stay $(P<0.05)$.

Significantly decreased enteric inflammation in low birth weight premature infants fed $B$. infantis EVC001 compared to infants born closer to term at the same gestational age. Inflammatory biomarker production, including cytokines and calprotectin, drives immunopathogenesis underlying the increased risk of morbidity and mortality in preterm infants [40,41]. Given our observational study design comparing EVC001-fed to no probiotic preterm infants, we first tested whether longitudinal cytokine and calprotectin concentrations were associated with clinical metadata or $B$. infantis EVC001 feeding using a multivariate analysis (Multivariate Analysis by Linear Models; MaAsLin, $\mathrm{n}=245)$. Here, we showed that total parenteral nutrition (TPN) usage $(\mathrm{mL} / \mathrm{kg} *$ day) significantly associated with increased IL-8 (Fig. 6a; 0.07 pg/mL, FDR-adjusted p value, $q=0.018$ ). Conversely, IL-1 $\beta$ and TNF $\alpha$

366 production decreased 0.064 and $0.1 \mathrm{pg} / \mathrm{mL}$ with increased TPN usage (mL/kg * day) (Fig. 6a; FDR-adjusted

367 p value, $q=0.018$ and 0.018 , respectively). Similarly, there was a weak negative relationship between

368 calprotectin, IL-1 $\beta$, IL-8, and TNF $\alpha$ concentration and age (in days) at a rate of 0.04, 0.07, 0.1, and 0.08 
$\mathrm{pg} / \mathrm{mL}$ per day, respectively (Fig. 6a; FDR-adjusted p value, $q=0.0001,0.0001,0.002$, and 0.0001,

370 respectively). Notably, the strongest relationship was observed between decreased TNF $\alpha$ production with

371 B. infantis EVC001 inclusion in the feeding protocol (Fig. 6a; $-1.06 \mathrm{pg} / \mathrm{mL}$, FDR-adjusted $p$ value, $q=$

372 0.01). Inhaled steroid usage, diaper rash, infant formula exposure, daily probiotic, birth weight (g), breast

373 milk consumption ( $\mathrm{mL} / \mathrm{kg}$ per day), antibiotic exposure at sampling, and gestational age at birth (weeks)

374 were not significantly associated with differences in cytokine and calprotectin concentrations when all other

375 clinical variables were considered (Fig. 6a). It is important to note the relationship between TPN and DOL

376 with cytokine and calprotectin concentrations were weak and the coefficient of the relationship was below

377 the level of detection for the assay.

378 Given the multivariate analysis identified B. infantis EVC001 feeding as the strongest association

379 with decreased enteric inflammation, we sought to corroborate whether there was an association of $B$.

380 infantis EVC001 with cytokine concentrations (IFN $\gamma$, IL-10, IL-13, IL-17A, IL-1 $\beta$, IL-2, IL-4 IL-5, IL-8,

381 and TNF $\alpha$ ). B. infantis absolute abundance was negatively correlated with the production of IL-8 (Fig. 6c;

$382 P=0.001, \rho=-0.24)$ independent of microbiome cluster characterization and marginally negatively

383 correlated with TNF $\alpha$, though the latter was not significant $(P=0.055 ; \rho=-0.14)$.

384 Gut microbiome composition has previously been shown to modulate the enteric inflammatory 385 markers in term and preterm infants $[10,16]$. Moreover, the hospital feeding protocol continued feeding of 386 B. infantis EVC001 up to 34 weeks cGA (see Methods). Given that other clinical differences between the 387 infants had small or nonsignificant associations with cytokine production, we next investigated whether 388 EVC001-fed preterm infants had an altered enteric inflammation profile compared to no probiotic group at 38934 weeks cGA. We selected samples collected from individual patients in each feeding group as close as 390 possible to 34 weeks cGA and compared the compositional changes in the microbiome at this time point 391 between the EVC001-fed ( $\mathrm{n}=29$; mean cGA 34.1 weeks) and no probiotics preterm infants $(\mathrm{n}=28$; mean 392 cGA 35.4 weeks). Among these samples, the only significantly different bacterial family was 393 Bifidobacteriaceae, which was more abundant among samples from B. infantis EVC001-fed infants in this 394 sampling window (FDR-corrected $P<0.0001$ ), though microbiome composition of the control group was 
substantially more variable between subjects (Supplementary Table 5). The composition of cluster identity of the samples identified in the 34-week cGA sampling window were significantly different between the two treatment groups $\left(\chi^{2} P<0.0001\right)$, with far more $S$. epidermidis cluster associated samples among infants who did not receive the probiotic, and more $B$. longum cluster associated samples among infants fed $B$. infantis EVC001 (Supplementary Table 5). When considering samples from infants nearest 34 weeks cGA,

400 significantly lower concentrations of calprotectin, IL-4, IL-5, IL-8, IL-17A, and TNF $\alpha$ (Fig. 6d-i; FDR401 corrected $P=0.009,0.039,0.0260 .009,0.032$, and 0.009 , respectively) were observed among infants fed 402 B. infantis EVC001 compared to infants not fed probiotics.

Taken together, these data showed that clinical metadata, including inhaled steroid use, diaper rash

404 incidence, infant formula, daily probiotic, birth weight, breast milk consumption, antibiotic usage, and most importantly gestational age were not associated with fecal cytokine or calprotectin concentration. However, the abundance of $B$. infantis EVC001, assessed by subspecies-specific qPCR, correlated with decreased enteric inflammation. Further, EVC001-fed preterm infants had significantly lower levels of key proinflammatory biomarkers compared to samples from infants not fed B. infantis EVC001, which suggests colonization of the preterm infant gut microbiome by B. infantis EVC001 may help modulate enteric inflammation in premature infants.

\section{Discussion}

413 The gut microbiome plays a major role in the development of the intestinal mucosa, including 414 maturation of physiological, anatomical, and biochemical functions in infants [12]. The intestinal 415 microbiome composition and the infant's response to these colonizing bacteria are important risk factors 416 for increased morbidity and mortality, including necrotizing enterocolitis (NEC) and sepsis in premature 417 infants [21,27,42-44]. Moreover, it is evident premature infants born in hospitalized environments are 418 colonized by nosocomially-acquired bacteria, including antibiotic-resistant bacteria [3]. This increases the 419 risk of serious infection and death [2] and presents deleterious implications for preterm infant growth and 420 development [45]. While human milk feeding is an important component of the current strategy to mitigate 
421 these effects, our findings show that it is insufficient, alone, to prevent colonization by nosocomial

422 pathogens or diminish the impact of these pathogens on the enteric immune system [16]. Therefore, the 423 importance of early microbiome development on host health and morbidity and the relative plasticity of

424 this community presents a need and an opportunity to colonize the preterm gut microbiome with beneficial 425 organisms that maximize ecosystem services to the host [23]. These services include increased availability 426 of nutrients from human milk, improved maturation of the intestinal epithelium, reduced enteric 427 inflammation, and mitigated risk of infection in hospitalized infants $[3,21,43,44,46]$. In this study, we found 428 preterm infants in two hospitals were colonized by nosocomially acquired bacteria despite a human milk 429 diet, and the resulting gut microbiomes, absent intervention with $B$. infantis EVC001, developed 430 comparably to reports in the literature on the preterm infant gut microbiome $[1,2,10]$. Early acquisition of 431 skin-derived bacteria (e.g. Staphylococcus epidermidis) and rapid colonization of the microbiome by 432 Enterobacteriaceae is consistent with examples of preterm infant gut microbiome development in the 433 literature [47,48], with taxa-dependent implications for preterm infant morbidity and development $434[4,10,45]$. Among preterm infants fed EVC001, we observed that novel microbiome compositions 435 developed, where colonization by $B$. infantis resulted in the displacement of bacteria associated with 436 increased risk of preterm morbidities [5](e.g. Fig. 2b-d, Fig. 3). Moreover, when preterm infants were fed 437 B. infantis EVC001, their overall microbiome was significantly enriched in functions required to access 438 HMOs [29] (Fig. 4). This is particularly relevant because the ability to utilize HMOs as prebiotic substrates 439 enables B. infantis EVC001 to thrive and modify the biochemical environment by the production of lactate 440 and acetate [24]. Furthermore, the consumption of HMOs by B. infantis, an infant-adapted Bifidobacterium, 441 produces critical immune modulatory metabolites, including indole-3-lactic acid [49]. This functional 442 adaptation to human milk also defines the symbiotic interaction between $B$. infantis and the human infant 443 and is key to the delivery of critical microbiome ecosystem services important to health and nutrition [23].

444 Epidemiological data have undeniably demonstrated that breastmilk confers benefits for early and lifelong 445 health to infants [50]. However, the infant also relies on the gut microbiome to fully realize the benefits 446 from breastmilk, especially HMOs. We found human milk feeding alone was not able to reduce the 
447 abundance of dysbiotic taxa (e.g. E. coli; Fig. 2a). In contrast, our results showed that feeding B. infantis

448 EVC001 was required to enrich the functions necessary to harvest additional energy from breastmilk and

449 limit populations of bacteria associated with dysbiosis and poor growth (e.g. Klebsiella, 450 Enterobacteriaceae) [45] (Fig. 2, Fig. 3).

451 Interestingly, we observed EVC001-fed preterm infants had significantly less antibiotic exposure

452 (Table 1) and significantly fewer incidences of diaper rash despite their relative prematurity at birth, which

453 warrants further investigation to understand whether this was directly or indirectly related to colonization

454 with $B$. infantis EVC001. Additionally, these infants had significantly fewer ARGs as a fraction of their

455 microbiome, with a total reduction in the resistome of $80.6 \%$ compared to no probiotic group. Specifically,

456 multidrug resistance genes were, on average, 227-fold higher in infants not fed a probiotic and some

457 samples had several thousand-fold higher abundance for specific ARGs compared to EVC001-fed infants,

458 including Mpha, which confers resistance to macrolide antibiotics, including erythromycin and

459 azithromycin [51]. The majority of the ARGs identified in this study are known to be present in potentially

460 pathogenic bacterial species, including Klebsiella and Enterobacter spp., which are responsible for

461 nosocomial infections as well as more severe morbidities including NEC [52,K 53] and late-onset sepsis

$462[52, \mathrm{~K} 53]$. In addition, we detected unique ARG signatures transferred to infants, and were able to observe

463 the rapid acquisition of site-specific ARGs, which highlights the NICU environment as a source of

464 antibiotic resistance microbes that can be transferred between NICUs [54]. We also found that infants

465 acquire these ARGs throughout their NICU stay, suggesting that reducing hospitalization times may limit

466 the acquisition of nosocomially acquired antibiotic-resistant bacteria. Importantly, targeted gut microbiome

467 modulation using $B$. infantis EVC001 has now been shown to be an effective strategy to combat the spread

468 of ARGs in both term [55] and preterm infants and is unlikely to lead to the development of novel resistance

469 mechanisms.

470 Key Bifidobacterium species have been associated with normal development of immune tolerance,

471 and B. infantis, in particular, has been shown to normalize the permeability of the intestinal mucosa [56,57],

472 which may partially explain why we saw a significant reduction in enteric cytokine production similar to 
473 previously reported findings in term infants [16]. Indeed, we observed that EVC001-fed preterm infants

474 had significantly lower enteric inflammation compared to preterm infants not fed a probiotic, which may

475 be due in part to the reduction in the abundance of potentially pathogenic bacteria associated with higher

476 levels of endotoxin [24]. Alternatively, the absolute abundance of B. infantis EVC001, independent of

477 microbiome composition, correlated with decreased proinflammatory cytokine profiles, which indicates a

478 benefit in feeding $B$. infantis EVC001 and may support the hypothesis that $B$. infantis-derived bacterial

479 metabolites, produced through the utilization of HMOs, induce mucosal immune tolerance in the gut

480 [49,58]. Notably, Bifidobacterium-derived acetate has previously been shown to mitigate the pathogenesis

481 of Enterobacteriaceae infection in an animal model [57], and B. infantis-derived indole-3-lactic acid

482 production reduced pathogen-induced inflammation in enterocytes in vitro $[49,58]$ through activation of

483 the aryl hydrocarbon receptor [49,59]. Recently, differences in B. infantis HMO utilization loci have been

484 identified [60], and strains missing key genes involved in HMO utilization are unlikely to confer the same

485 benefits to infants as we observed here, as the conversion of indigestible HMOs to infant accessible short

486 chain fatty acids and indole-3-lactic acid are key functions of a healthy gut microbiome, whether in term or

487 preterm infants [23].

There are some limitations to the results presented here, chiefly by the observational design of the study. Equivalent longitudinal data sets from both cohorts, those not receiving probiotics and those fed $B$. infantis EVC001, at similar cGAs were not available with which to compare our findings throughout the 491 duration of their hospital stays. Given these potential confounding variables in our data set, we compared 492 the effect of those differences in clinical variables on the gut microbiome and cytokine profile using 493 MaAsLin, between EVC001-fed infants and infants who were not fed a probiotic (Fig. 2a, 6a). We found 494 that these differences in gestational age or weight at birth are unlikely to be responsible for the significant 495 changes we observed between the feeding cohorts, given the strongest effect was related to probiotic 496 introduction, and the abundance of the probiotic organism (i.e. B. infantis) was linked to diminished 497 abundance of taxa associated with preterm neonatal morbidities (Fig. 2), with increased capacity for HMO 498 utilization (Fig. 4), reduced ARG abundance (Fig. 5), and with diminished enteric inflammation (Fig. 6). 


\section{Conclusions}

Overall, the detailed genomic gut microbiome and fecal inflammatory biomarker observations

501 presented here demonstrate that preterm gut microbiome composition can be altered by feeding $B$. infantis

502 EVC001. Using multivariate modeling we found that, independent of clinical variables associated with

503 degree of prematurity or hospital care, these changes were uniquely related to feeding B. infantis EVC001

504 and that this measure had a significant and beneficial impact on the infant's enteric inflammatory profile.

505 We conclude that the use of $B$. infantis EVC001 in conjunction with human milk in premature infants

506 provides a meaningful and low-risk approach to alter the gut microbiome composition and increase the

507 abundance of a well-established infant gut symbiont that (1) increased human milk utilization, (2)

508 diminished enteric inflammation, and (3) decreased the abundance of taxa associated with antibiotic-

509 resistance and diminished health outcomes [45]. Together with human milk feeding, B. infantis EVC001

510 may help to mitigate microbiome-associated risks of morbidity and mortality in hospitalized infants. Future

511 observational and/or controlled studies examining the impact of $B$. infantis EVC001 on preterm infant

512 health outcomes are warranted.

\section{Study Design}

517 This study was performed at Kaiser Orange County Anaheim and Irvine Neonatal Intensive Care Units 518 (NICUs) under oversight from the Institutional Review Board. Eligible preterm infants were enrolled 519 during the period of May 2019 to October 2019. Inclusion criteria included premature birth less than 39 520 weeks corrected gestational age (cGA) or less than 1500 grams. At the time of stool collection, site

521 personnel recorded data about the infants and the stool samples and this information was cross-verified by 522 independent hospital staff by comparing de-identified subject and sample metadata from electronic medical 523 records. Data collected from collection logs and electronic medical records included antibiotic exposure 524 on collection days, stool type (meconium, non-meconium, mixed), subject's gestational age at birth, 525 corrected gestational age at sample collection, subject's intake of probiotics, medication, and subject's 
current diet (ie. human milk and/or infant formula). Stool samples tubes and stool collection log entry was

527 tagged with linked barcoded labels by the study personnel. Fecal samples were collected from the diaper 528 by site personnel, transferred into sterile collection tubes, and immediately frozen in a designated freezer (529 20C) inside the NICU. Stool samples were collected weekly and shipped on dry ice to the laboratory where 530 they were aliquoted and stored at $-80 \mathrm{C}$ until analysis. A total of 298 fecal samples from 77 premature 531 infants throughout 26-40 weeks corrected gestational age were collected from Anaheim and Irvine NICUs.

\section{Bacterial DNA Methods}

534 DNA was extracted from 296 stool samples aliquots stored in DNA/RNA shield lysis 535 tubes (Zymo Research, Irvine CA) using the ZymoBIOMICS 96 MagBead DNA kit (Zymo Research). 536 Extracted DNA was quantified using QuantIT dsDNA Assay kit, high sensitivity 537 (ThermoFisher Scientific, Waltham, MA) according to the manufacturer's protocol. Three (3) samples were omitted from downstream analysis due to failure to meet input requirements for library preparation.

539 Libraries were prepared for each sample using the Illumina Nextera DNA Flex library kit (Illumina, San

540 Diego, CA) with unique dual indexes according to manufacturer guidelines. Libraries were pooled and 541 submitted to UC Davis DNA Technologies Core for sequencing on an Illumina NovaSeq S4 542 flow cell. (Illumina, San Diego, CA). Each lane of the S4 flow cell contained 96 libraries.

\section{Absolute quantification of B. infantis by Quantitative Real-Time PCR}

545 Quantification of the total B. infantis was performed by quantitative real-time PCR using Blon_2348 546 sialidase gene primers Inf2348F (5'- ATA CAG CAG AAC CTT GGC CT -3' ), Inf2348_R (5'- GCG ATC 547 ACA TGG ACG AGA AC -3' ), and Inf2348_P (5'- /56-FAM/TTT CAC GGA /ZEN/TCA CCG GAC CAT 548 ACG /31ABkFQ/-3') [61]. Each reaction contained $10 \mu \mathrm{L}$ of $2 \times$ TaqMan Universal Master Mix II with UNG 549 master mix (ThermoFisher Scientific, Waltham, MA), $0.9 \mu \mathrm{M}$ of each primer, $0.25 \mu \mathrm{M}$ probe and $5 \mu \mathrm{L}$ of 550 template DNA. Thermal cycling was performed on a QuantStudio 3 Real-Time PCR System and consisted 551 of an initial UNG activation step of 2 minutes at $50^{\circ} \mathrm{C}$ followed by a 10 minute denaturation at $95^{\circ} \mathrm{C}$, 
552 succeeded by 40 cycles of $15 \mathrm{~s}$ at $95^{\circ} \mathrm{C}$ and $1 \mathrm{~min}$ at $60^{\circ} \mathrm{C}$. Standard curves for absolute quantification were 553 generated using genomic DNA extracted from a pure culture of B. infantis EVC001.

\section{Quality filtering and removal of human sequences}

555 Demultiplexed fastq sequences were quality filtered, including adaptor trimming using Trimmomatic v0.36

556 [62] with default parameters. Quality-filtered sequences were screened to remove human sequences using

557 GenCoF v1.0 [28] against a non-redundant version of the Genome Reference Consortium Human Build 38,

558 patch release 7 (GRCh38_p7; www.ncbi.nlm.nih.gov). Human sequence-filtered raw reads were deposited

559 in the Sequence Read Archive (SRA; https://www.ncbi.nlm.nih. gov/sra) under the reference number,

560 PRJNA630999.

\section{Taxonomic and strain profiling}

562 Taxonomic profiling of the metagenomic samples was performed using MetaPhlAn2 [63], which uses a

563 library of clade-specific markers to provide pan microbial (bacterial, archaeal, viral, and eukaryotic)

564 profiling (http://hutten- hower.sph.harvard.edu/metaphlan2), in combination with Humann2 [64] to profile

565 functional metagenomics against Uniref90 following the updated global profiling of the Human

566 Microbiome Project (2017) [65]. Cross-database annotations (e.g., UniProt to KEGG) were performed

567 within Humman2 using the "utility_mapping” conversion tool package.

\section{Taxonomic cluster grouping assignment}

569 Species-level taxonomic data consisting of 218 species relative abundance across 292 samples and 77 570 subjects was used for the analysis. Following the methodology from a previously published study [66], 571 samples were clustered using Jensen-Shannon Divergence (JSD) distance and the Partitioning Around 572 Mediods clustering algorithm. The results were assessed for the optimal number of clusters using the 573 Calinski-Harabasz $(\mathrm{CH})$ Index and Silhouette coefficient. Clustering performed on the full dataset did not

574 show a clear optimal number of clusters. Additionally, uneven sampling between subjects could lead to 575 cluster formation dominated by one or a few subjects with a high number of samples. Because of these 576 concerns, subsetted datasets were created by randomly selecting one sample per subject 1000 times. Each 577 of the subsetted datasets were clustered with the same methodology as the full dataset. $\mathrm{CH}$ index was 
computed for iterations from 2 to 6 clusters within each subset. The maximum of those values was chosen

579 as the optimal number of clusters for that subset. Maximum $\mathrm{CH}$ index coefficients were stored and observed

580 after the 1000 cluster iterations and, again, did not show a clear choice for an optimal number of clusters.

581 Instead, between-class analysis (BCA) was used to identify drivers for clusters. The top $n$ important species

582 (where $\mathrm{n}=$ twice the number of clusters) were saved for each iteration. Top important taxa were then

583 associated with a specific cluster by examining the angle of importance with the angle of the cluster median

584 on the first two component axes. If the angle was within $+/-22.5$ degrees, that species was considered to

585 be associated with that cluster. Any species shown both as important and associated with a specific cluster

586 at least 800 times over the 1000 iterations was considered a primary driver. Four species $(E$. coli,

587 unclassified Escherichia species, B. longum species, and S epidermidis) met these criteria. Enterotyping of

588 subsetted datasets proved challenging and inconclusive, therefore these datasets were further restricted to

589 include only the top cluster-driving bacteria. The $\mathrm{CH}$ index and silhouette were examined on the species-

590 restricted datasets and both metrics pointed to 3 as the optimal number of clusters. BCA was performed

591 again on the species-restricted data and showed a pattern of B. longum species, S. epidermidis and E.

592 coli/unclassified Escherichia clusters. The cluster that each sample fell in over the 1000 iterations was

593 examined. Most samples fell within the same species-driven cluster $100 \%$ of the time and all samples fell

594 within the same cluster at least $90 \%$ of the time. Confident that this method of clustering was robust, each

595 sample was then assigned to the cluster it fell into most frequently.

\section{Antibiotic resistance gene analysis}

597 We applied ShortBRED [67,68] to profile antibiotic resistance (AR) abundance and composition in 598 the infant gut microbiome. We first produced a set of new AR marker sequences by applying ShortBRED599 Identify to the Comprehensive Antibiotic Resistance Database database (CARD) [69].

600 We then used ShortBRED-Quantify to profile the relative abundance of corresponding antibiotic resistance 601 genes (ARGs). Final values were normalized in Reads Per Kilobase per Million mapped reads (RPKM) to 602 account for sequencing depth as well as gene length. We used custom scripts to collapse CARD individual 
antibiotic resistance gene entries in their corresponding drug class. Conversion rules are offered within

604 every CARD package update.

\section{Multiplexed Immunoassays}

606 Interleukin (IL)-1 $\beta$, IL-2, IL-4 IL-5, IL-8, IL-10, IL-13, IL-17A, interferon (IFN) $\gamma$, and tumor necrosis

607 factor (TNF) $\alpha$ were quantified from $80 \mathrm{mg}$ of frozen stool diluted 1:10 in Meso Scale Discovery (MSD;

608 Rockville, MD) diluent using the U-PLEX Inflammation Panel 1 (human) Kit and concentration of fecal

609 calprotectin was quantified using MSD R-PLEX Human Calprotectin Antibody Set.Standards and samples

610 were measured in duplicate and blank values were subtracted from all readings according to the

611 manufacturer's instructions as previously published [16]. All detectable biomarker values were included

612 as continuous data in the analyses; however, values below level of detection $(<30 \%$ of all cytokines and

613 calprotectin measurements) were generated below the level of quantification to justify parametric statistics.

614 Fecal cytokine and calprotectin concentrations were determined using calibrations curves to which

615 electrochemiluminescence signals were backfitted. Final concentrations were calculated using the Sector

616 Imager 2400 MSD Discovery Workbench analysis software.

\section{Statistical Analysis}

618 Statistical analyses were performed in R v3.6.2. A Kruskal-Wallis one-way analysis of variance

619 coupled with an FDR or Bonferroni correction was used for statistical comparisons between gene

620 groups, cytokines and taxa amongst groups. A post-hoc Dunn test was applied to determine

621 differences between species clusters. Statistical analysis to assess total resistome or enterotype

622 composition by group was performed using a Mann-Whitney or Holm-adjusted Dunn's test.

623 Rarefaction curves were computed to estimate the diversity of the identified ARGs across

624 samples. A nonparametric two-sample t-test was used to compare rarefaction curves using Monte

625 Carlo permutations ( $\mathrm{n}=999)$. To test for association of microbial abundance with clinical

626 metadata we performed a multivariate analysis using Multivariate Analysis by Linear Models

627 (MaAsLin2) version for R. Maaslin2 performs boosted, additive general linear models between 
628 metadata and microbial abundance (at the taxa level). Boosting of metadata and selection of a

629 model was performed per taxon and, separately, per inflammatory biomarker. The metadata used

630 were total parenteral nutrition (TPN; $\mathrm{mL} / \mathrm{kg}$ per day), inhaled steroid use associated with sample

631 collection, diaper rash treatment/incidence (e.g. desitin), gestational age at birth (GA, in weeks),

632 formula use associated with sampling, age in days (day of life, DOL), probiotic feeding protocol

633 (EVC001-fed or no probiotic), birth weight (grams), human milk consumption (mL/kg per day),

634 and antibiotic exposure associated with sampling. A linear mixed effects model was used to

635 examine the effects of probiotic use on ARG acquisition. ARG counts per sample were modeled

636 with probiotic use, day of life and hospital as fixed effects and hospital and subject as random

637 effects. For random effects, we also examined the degree to which the hospital effect on a given

638 subject deviates from the global effect of hospital. $\chi^{2}$ tests were used to compare species cluster

639 compositions between groups, where indicated. Cytokines and B. infantis-specific qPCR were

640 correlated with the Spearman method with FDR correction. $P$ values throughout the manuscript

641 are represented by asterisks (****, $\left.P<0.0001 ; * * *, P<0.001 ;{ }^{* *}, P<0.01 ; *, P<0.05\right)$. 


\section{Ethics approval and consent to participate}

646 The study protocol for preterm infants was approved by the Institutional Review Board, Kaiser Permanente

647 Southern California (\#12079). Preterm participants were recruited from the neonatal intensive care unit at

648 Kaiser Permanente Southern California, Irvine and Anaheim, California between May 2019 to October

649 2019. Inclusion criteria included premature birth less than 39 weeks corrected gestational age (cGA) or less

650 than 1500 grams. Parental informed consent was given.

651

652

653

654

655

656

657

658

659

660

661

662

663

664

665

666

667

668

669

670

671

\section{Consent for publication}

Not applicable

\section{Availability of data and material}

We are committed to making our data, materials, and analysis methods open and available upon request, where permitted. Data generated in this study including human sequence-filtered raw reads are deposited in the Sequence Read Archive (SRA; https://www.ncbi.nlm.nih.gov/sra) under the reference number, PRJNA630999. qPCR and inflammatory marker raw data is attached to be available as Source Data. The code of 'Clustering of Microbiome Metagenomics' validation model is available online through the permanent repository at https:/github.com/hbrown-evolvebiosystems/Cluster-Validation-Algorithm. The R packages used are listed here: readr, readxl, tidyverse, lme4, car, MASS, pscl, sjPlot, sjlabelled, sjmisc, sjstats, viridis, scales, lattice, patchwork, gridExtra, devtools, BlocManager, installr, cluster, clusterSim, ade4, ggpubr, psych, dunn.test, wesanderson, matlib, data.table, rstatix.

\section{Competing interests}

MN, HH, PM, MAA, SE, MG, and C-GO are employees of Southern California Kaiser Permanente, a not for profit healthcare system. CMG, CBH, RDM, SK, SC, GC, HKB, SAF, and BMH are employees of Evolve Biosystems, a company focused on restoring the infant microbiome. $\mathrm{HH}$ is a member of the Evolve Biosystems Clinical Advisory Board. SAF and BMH serve as Adjunct Assistant Professors in Food Science \& Technology Department, University of Nebraska Lincoln. 
674 This work was supported by Evolve Biosystems.

675

676

Authors' contributions

$677 \mathrm{BMH}, \mathrm{MN}$, and $\mathrm{HH}$ conceived the study. BMH, MN, HH, PM, MAA, SAF, CM, and CBH designed the

678 study. SE, MG, CGO, CM, collected samples analyzed in this study. RDM, SC, and GC generated data

679 presented in the manuscript. RDM, SK, SC, GC, HKB, SAF, and BMH analyzed the data. MN, GC, SAF,

680 and BMH wrote the manuscript. All authors approved the final manuscript for publication.

681

682 Acknowledgements

683 The authors thank the nurses and clinical staff of Southern Kaiser Neonatal Intensive Care Units at Anaheim

684 and Irvine for their tireless collection of infant samples.

685

686

687

Author Information

${ }^{1}$ Neonatology, Kaiser Permanente Orange County, Anaheim, CA, United States; ${ }^{2}$ Kaiser Permanente

688 Medicine, United States; ${ }^{3}$ Evolve Biosystems Inc., Davis, CA, United States; ${ }^{4}$ Department of Food Science

689 and Technology, University of Nebraska Lincoln; Lincoln, NE United States.

690

691

692

693 
695 1. Groer MW, Luciano AA, Dishaw LJ, Ashmeade TL, Miller E, Gilbert JA. Development of the preterm infant gut microbiome: a research priority. Microbiome. BioMed Central; 2014;2:38-8.

697 2. Grier A, Qiu X, Bandyopadhyay S, Holden-Wiltse J, Kessler HA, Gill AL, et al. Impact of prematurity and nutrition on the developing gut microbiome and preterm infant growth. Microbiome; 2017;1-19.

3. Brooks B, Olm MR, Firek BA, Baker R, Thomas BC, Morowitz MJ, et al. Strain-resolved analysis of hospital rooms and infants reveals overlap between the human and room microbiome. Nature Communications. Nature Publishing Group; 2017;8:1814.

4. Pammi M, Cope J, Tarr PI, Warner BB, Morrow AL, Mai V, et al. Intestinal dysbiosis in preterm infants preceding necrotizing enterocolitis: a systematic review and meta-analysis. Microbiome; 2017:115.

5. Dobbler PT, Procianoy RS, Mai V, Silveira RC, Corso AL, Rojas BS, et al. Low Microbial Diversity and Abnormal Microbial Succession Is Associated with Necrotizing Enterocolitis in Preterm Infants. Front. Microbiol. Frontiers; 2017;8:583-12.

6. Stewart CJ, Embleton ND, Marrs ECL, Smith DP, Fofanova T, Nelson A, et al. Longitudinal development of the gut microbiome and metabolome in preterm neonates with late onset sepsis and healthy controls. Microbiome; 2017;1-11.

714 8. Warner BB, Deych E, Zhou Y, Hall-Moore C, Weinstock GM, Sodergren E, et al. Gut bacteria

7. Mai V, Torrazza RM, Ukhanova M, Wang X, Sun Y, Li N, et al. Distortions in Development of Intestinal Microbiota Associated with Late Onset Sepsis in Preterm Infants. Denning PW, editor. PLoS ONE. 2013;8:e52876-9. dysbiosis and necrotising enterocolitis in very low birthweight infants: a prospective case-control study. The Lancet. 2016;387:1928-36.

9. Shin N-R, Whon TW, Bae J-W. Proteobacteria: microbial signature of dysbiosis in gut microbiota. Trends in Biotechnology. Elsevier Ltd; 2019;1-8.

10. Ho TTB, Groer MW, Kane B, Yee AL, Torres BA, Gilbert JA, et al. Enteric dysbiosis and fecal calprotectin expression in premature infants. Pediatric Research. Springer US; 2019:1-8.

11. Ford H, Watkins S, Reblock K, surgery MRJOP, 1997. The role of inflammatory cytokines and nitric

722 oxide in the pathogenesis of necrotizing enterocolitis. Elsevier

723 12. Sharma R, Tepas JJ III. Microecology, intestinal epithelial barrier and necrotizing enterocolitis.

724 Pediatr. Surg. Int. 2009;26:1635-19.

725 13. Emami CN, Chokshi N, Wang J, Hunter C, Guner Y, Goth K, et al. Role of interleukin-10 in the

726 pathogenesis of necrotizing enterocolitis. AJS. Elsevier Inc; 2012;203:428-35.

727 14. Claud EC, Savidge T, Walker WA. Modulation of human intestinal epithelial cell IL-8 secretion by

728 human milk factors. Pediatr. Res. 2003;53:419-25. 
15. Alcon-Giner C, Dalby MJ, Caim S, Ketskemety J, Shaw A, Sim K, et al. Microbiota Supplementation with Bifidobacterium and Lactobacillus Modifies the Preterm Infant Gut Microbiota and Metabolome: An Observational Study. Cell Reports Medicine. ElsevierCompany; 2020;1:100077.

16. Henrick BM, Chew S, Casaburi G, Brown HK, Frese SA, Zhou Y, et al. Colonization by B. infantis EVC001 modulates enteric inflammation in exclusively breastfed infants. Pediatric Research. Springer US; 2019:1-9.

17. Fleming P, Wilks M, Eaton S, Panton N, Hutchinson R, Akyempon A, et al. Exploratory Studies from the PiPS Trial fail to find evidence that Bifidobacterium breve BBG-001 modifies intestinal barrier function. Pediatric Research. Springer US; 2020:1-0.

18. Hoyos AB. Reduced incidence of necrotizing enterocolitis associated with enteral administration of Lactobacillus acidophilus and Bifidobacterium infantis to neonates in an intensive care unit. International Journal of Infectious Diseases. Elsevier; 1999;3:197-202.

19. AlFaleh K, Anabrees J. Probiotics for prevention of necrotizing enterocolitis in preterm infants. Evid.Based Child Health. 2014;9:584-671.

20. van den Akker CHP, van Goudoever JB, Shamir R, Domellöf M, Embleton ND, Hojsak I, et al. Probiotics and Preterm Infants. Journal of Pediatric Gastroenterology and Nutrition. 2020:1-66.

21. Underwood MA, Arriola J, Gerber CW, Kaveti A, Kalanetra KM, Kananurak A, et al. Bifidobacterium longum subsp. infantis in experimental necrotizing enterocolitis: alterations in inflammation, innate immune response and the microbiota. Pediatr. Res. 2014;76:326-33.

22. Caplan MS, Miller-Catchpole R, Kaup S, Russell T, Lickerman M, Amer M, et al. Bifidobacterial supplementation reduces the incidence of necrotizing enterocolitis in a neonatal rat model. Gastroenterology. 1999;117:577-83.

23. Duar RM, Henrick BM, Casaburi G, Frese SA. Integrating the Ecosystem Services Framework to Define Dysbiosis of the Breastfed Infant Gut: The Role of B. infantis and Human Milk Oligosaccharides. Front. Nutr. Frontiers; 2020;7.

24. Frese SA, Hutton AA, Contreras LN, Shaw CA, Palumbo MC, Casaburi G, et al. Persistence of Supplemented Bifidobacterium longumsubsp. infantisEVC001 in Breastfed Infants. Krajmalnik-Brown R, editor. mSphere. 2017;2:e0501-17-15.

25. Casaburi G, Frese SA. Colonization of breastfed infants by Bifidobacterium longum subsp. infantis EVC001 reduces virulence gene abundance. Human Microbiome Journal. Elsevier; 2018;9:7-10.

26. Karav S, Casaburi G, Frese SA. Reduced colonic mucin degradation in breastfed infants colonized by Bifidobacterium longum subsp. infantis EVC001. FEBS Open Bio. John Wiley \& Sons, Ltd; 2018;8:1649-57.

27. Underwood MA, Kalanetra KM, Bokulich NA, Lewis ZT, Mirmiran M, Tancredi DJ, et al. A Comparison of Two Probiotic Strains of Bifidobacteria in Premature Infants. The Journal of Pediatrics. 2013;163:1585-9.

28. Czajkowski MD, Vance DP, Frese SA, 2019. GenCoF: a graphical user interface to rapidly remove human genome contaminants from metagenomic datasets. Bioinformatics. 
29. Sela DA, Chapman J, Adeuya A, Kim JH, Chen F, Whitehead TR, et al. The genome sequence of Bifidobacterium longum subsp. infantis reveals adaptations for milk utilization within the infant microbiome. Proceedings of the National Academy of Sciences. National Acad Sciences; 2008;105:18964-9.

30. Garrido D, Kim JH, German JB, Raybould HE, Mills DA. Oligosaccharide Binding Proteins from

Bifidobacterium longum subsp. infantis Reveal a Preference for Host Glycans. Uversky V, editor. PLoS ONE. 2011;6:e17315-13.

31. Turroni F, Bottacini F, Foroni E, Mulder I, Kim JH, Zomer A, et al. Genome analysis of Bifidobacterium bifidum PRL2010 reveals metabolic pathways for host-derived glycan foraging. Proceedings of the National Academy of Sciences. National Academy of Sciences; 2010;107:19514-9.

32. Egan M, Motherway MOC, Kilcoyne M, Kane M, Joshi L, Ventura M, et al. Cross-feeding by Bifidobacterium breve UCC2003 during co-cultivation with Bifidobacterium bifidum PRL2010 in a mucin-based medium. 2014;1-14.

33. Smilowitz JT, O'Sullivan A, Barile D, German JB, Lönnerdal B, Slupsky CM. The Human Milk Metabolome Reveals Diverse Oligosaccharide Profiles. Journal of Nutrition. 2013;143:1709-18.

34. Krumbeck JA, Maldonado-Gomez MX, Ramer-Tait AE, Hutkins RW. Prebiotics and synbiotics. Curr. Opin. Gastroenterol. 2016;32:110-9.

35. Awad A, Arafat N, Elhadidy M. Genetic elements associated with antimicrobial resistance among avian pathogenic Escherichia coli. Annals of Clinical Microbiology and Antimicrobials. BioMed Central; 2016;1-8.

36. Ramirez MS, Nikolaidis N, Tolmasky ME. Rise and dissemination of aminoglycoside resistance: the aac(6')-Ib paradigm. Front. Microbiol. Frontiers; 2013;4:121.

37. Friedman L, Alder JD, Silverman JA. Genetic changes that correlate with reduced susceptibility to daptomycin in Staphylococcus aureus. Antimicrob. Agents Chemother. American Society for Microbiology Journals; 2006;50:2137-45.

38. Bhakta M, Bal M. Identification and Characterization of a Shuttle Plasmid with Antibiotic Resistance Gene from Staphylococcus aureus. Current Microbiology. 2003;46:413-7.

39. Schwarz FV, Perreten V, Teuber M. Sequence of the 50-kb Conjugative Multiresistance Plasmid pRE25 from Enterococcus faecalis RE25. Plasmid. 2001;46:170-87.

40. Maheshwari A, Schelonka RL, Dimmitt RA, Carlo WA, Munoz-Hernandez B, Das A, et al. Cytokines associated with necrotizing enterocolitis in extremely-low-birth-weight infants. Pediatric Research. 2014;76:100-8.

41. Benkoe T, Reck C, Pones M, Weninger M, Gleiss A, Stift A, et al. Interleukin-8 predicts 60-day mortality in premature infants with necrotizing enterocolitis. J. Pediatr. Surg. Elsevier Inc; 2014;49:3859.

42. Milani C, Duranti S, Bottacini F, Casey E, Turroni F, Mahony J, et al. The First Microbial Colonizers of the Human Gut: Composition, Activities, and Health Implications of the Infant Gut Microbiota. Microbiology and Molecular Biology Reviews. 2017;81:1-67. 
43. Bode L, Raman AS, Murch SH, Rollins NC, Gordon JI. Understanding the mother-breastmilk-infant "triad." Science. American Association for the Advancement of Science; 2020;367:1070-2.

44. Younge NE, Newgard CB, Cotten CM, Goldberg RN, Muehlbauer MJ, Bain JR, et al. Disrupted Maturation of the Microbiota and Metabolome among Extremely Preterm Infants with Postnatal Growth Failure. Sci Rep. 2019;9:8167.

810 45. Yee AL, Miller E, Dishaw LJ, Gordon JM, Ji M, Dutra S, et al. Longitudinal Microbiome Composition and Stability Correlate with Increased Weight and Length of Very-Low-Birth-Weight Infants. Whiteson KL, editor. mSystems. American Society for Microbiology Journals; 2019;4:428-12. Infants: Immunological Modulation and Implication in Neonatal Outcomes. Front Immunol. 2019;10:641-11.

47. Gibson MK, Bin Wang, Ahmadi S, Burnham C-AD, Tarr PI, Warner BB, et al. Developmental dynamics of the preterm infant gut microbiota and antibiotic resistome. Nature Microbiology. Nature Publishing Group; 2016;1:1-10

48. Romano-Keeler J, Moore DJ, Wang C, Brucker RM, Fonnesbeck C, Slaughter JC, et al. Early life establishment of site-specific microbial communities in the gut. Gut Microbes. 2014;5:192-201.

49. Ehrlich AM, Henrick B, Pacheco A, Taft D, Xu G, Huda N, et al. Bifidobacterium grown on human milk oligosaccharides produce tryptophan metabolite Indole-3-lactic acid that significantly decreases inflammation in intestinal cells in vitro. FASEB J. Federation of American Societies for Experimental Biology; 2018;32:lb359-9.

50. MD PCGV, MD RB, MD PAJDB, PhD GVAF, PhD PSH, MSc JK, et al. Series Breastfeeding 1 Breastfeeding in the 21st century: epidemiology, mechanisms, and lifelong effect. The Lancet. Elsevier Ltd; 2016;387:475-90.

51. Ericson JE, Arnold C, Cheeseman J, Cho J, Kaneko S, Wilson E, et al. Use and Safety of Erythromycin and Metoclopramide in Hospitalized Infants. Journal of Pediatric Gastroenterology and Nutrition. 2015;61:334-9.

52. Sim K, Shaw AG, Randell P, Cox MJ, McClure ZE, Li MS, et al. Dysbiosis Anticipating Necrotizing Enterocolitis in Very Premature Infants. Clin. Infect. Dis. 2015;60:389-97.

53. Kangozhinova K, Abentayeva B, Repa A, Baltabayeva A, Erwa W, Stauffer F. Kulturmäßig bewiesene Neugeborenensepsis mit besonderer Berücksichtigung der durch Enterobacteriaceae hervorgerufenen late-onset Sepsis in einer Neugeborenen Station (Level 3) in Astana, Kasachstan. Wien Klin Wochenschr. 2013;125:611-5.

54. Ramirez CB, Cantey JB. Antibiotic Resistance in the Neonatal Intensive Care Unit. Neoreviews. 2019;20:e135-44. modulation reduces the abundance of antibiotic- resistant bacteria. Antimicrobial Resistance \& Infection Control; 2019:1-18. 
56. Chichlowski M, De Lartigue G, German JB, Raybould HE, Mills DA. Bifidobacteria Isolated From Infants and Cultured on Human Milk Oligosaccharides Affect Intestinal Epithelial Function. Journal of Pediatric Gastroenterology and Nutrition. 2012;55:321-7.

57. Fukuda S, Toh H, Hase K, Oshima K, Nakanishi Y, Yoshimura K, et al. Bifidobacteria can protect from enteropathogenic infection through production of acetate. Nature. 2011;469:543-7.

58. Di Meng, Sommella E, Salviati E, Pietro Campiglia, Ganguli K, Djebali K, et al. Indole-3-lactic acid, a metabolite of tryptophan, secreted by Bifidobacterium longum subspecies infantis is anti-inflammatory in the immature intestine. Pediatric Research. Springer US; 2020:1-9.

59. Laursen MF, Sakanaka M, Burg von N, Andersen D, Mörbe U, Rivollier A, et al. Breastmilkpromoted bifidobacteria produce aromatic lactic acids in the infant gut. 2020;387:475-38.

60. Albert K, Rani A, Sela DA. Comparative Pangenomics of the Mammalian Gut Commensal Bifidobacterium longum. Microorganisms. Multidisciplinary Digital Publishing Institute; 2020;8:7-22.

61. Lawley B, Munro K, Hughes A, Hodgkinson AJ, Prosser CG, Lowry D, et al. Differentiation of Bifidobacterium longumsubspecies longumand infantisby quantitative PCR using functional gene targets. PeerJ. PeerJ Inc; 2017;5:e3375-14.

62. Bolger AM, Lohse M, Bioinformatics BU, 2014. Trimmomatic: a flexible trimmer for Illumina sequence data. Bioinformatics. 2014.

63. Liang X-H, Shen W, Sun H, Migawa MT, Vickers TA, Crooke ST. Translation efficiency of mRNAs is increased by antisense oligonucleotides targeting upstream open reading frames. Nat. Biotechnol. 2016;34:875-80.

64. Franzosa EA, McIver LJ, Rahnavard G, Thompson LR, Schirmer M, Weingart G, et al. Species-level functional profiling of metagenomes and metatranscriptomes. Nat. Methods. Springer US; 2018;:1-12.

65. Lloyd-Price J, Mahurkar A, Rahnavard G, Crabtree J, Orvis J, Hall AB, et al. Strains, functions and dynamics in the expanded Human Microbiome Project. Nature Publishing Group. Nature Publishing Group; 2017:1-25.

66. MetaHIT Consortium (additional members), Arumugam M, Raes J, Pelletier E, Le Paslier D, Yamada T, et al. Enterotypes of the human gut microbiome. Nature. 2011;473:174-80.

67. Jakobsson HE, Jernberg C, Andersson AF, Sjölund-Karlsson M, Jansson JK, Engstrand L. ShortTerm Antibiotic Treatment Has Differing Long-Term Impacts on the Human Throat and Gut Microbiome. Ratner AJ, editor. PLoS ONE. Public Library of Science; 2010;5:e9836-12.

68. Kaminski J, Gibson MK, Franzosa EA, Segata N, Dantas G, Huttenhower C. High-Specificity Targeted Functional Profiling in Microbial Communities with ShortBRED. Noble WS, editor. PLoS Comput. Biol. 2015;11:e1004557-22.

69. Alcock BP, Raphenya AR, Lau TTY, Tsang KK, Bouchard M, Edalatmand A, et al. CARD 2020: antibiotic resistome surveillance with the comprehensive antibiotic resistance database. Nucleic Acids Res. Oxford University Press; 2019;10:226-9. 
Table 1. Characteristics of preterm infant feeding cohort and sample collection

\begin{tabular}{|c|c|c|c|c|}
\hline $\begin{array}{l}\text { Sample and Patient } \\
\text { Demographics }\end{array}$ & No Probiotic & B. infantis EVC001 & $P$ value & Statistical Comparison \\
\hline Total & 46 & 31 & & \\
\hline Female, $\mathrm{n}(\%)$ & $23(50)$ & $10(32)$ & 0.1651 & Fisher's Exact test \\
\hline Gestational age, wk, mean (SD) & $34.9(2.74)$ & $28.3(2.77)$ & $<0.001$ & Mann-Whitney U Test \\
\hline Birth weight, g, mean (SD) & $2207(738)$ & $1112(340)$ & $<0.001$ & Mann-Whitney U Test \\
\hline Caesearean delivery, $\mathrm{n}(\%)$ & $31(67)$ & $20(65)$ & 1 & Fisher's Exact test \\
\hline Fecal sample collected $n$ : & 104 & 193 & & \\
\hline $\begin{array}{r}\text { Specimes collected per baby, } \\
\text { mean (SD) }\end{array}$ & $2.26(1.71)$ & $6.23(3.35)$ & $<0.001$ & Mann-Whitney U Test \\
\hline $\begin{array}{r}\text { Antibiotic exposure within } 7 \text { days } \\
\text { of sampling, } n(\%)\end{array}$ & $14(13.5)$ & $10(5.2)$ & 0.024 & Fisher's Exact test \\
\hline $\begin{array}{r}\text { Infant formula use coinciding with } \\
\text { sampling, } \mathrm{n}(\%)\end{array}$ & $39(37.5)$ & $9(4.66)$ & $<0.001$ & Fisher's Exact test \\
\hline $\begin{array}{r}\text { Diaper rash reported coinciding } \\
\text { with sampling, } \mathrm{n}(\%)\end{array}$ & $14(13.5)$ & $10(5.2)$ & 0.024 & Fisher's Exact test \\
\hline $\begin{array}{r}\text { Steroid use coinciding with } \\
\text { sampling, } \mathrm{n}(\%)\end{array}$ & $1(0.96)$ & $43(22.28)$ & $<0.001$ & Fisher's Exact test \\
\hline $\begin{array}{l}\text { Fecal sample sequencing } \\
\text { passing quality filter, } n(\%)\end{array}$ & $101(97)$ & $191(99)$ & & \\
\hline $\begin{array}{r}\text { Fecal sample inflammatory } \\
\text { quantification, } \mathrm{n}(\%)\end{array}$ & $83(80)$ & $171(89)$ & & \\
\hline
\end{tabular}

\section{$881 \quad$ Figure Legends}

882 Fig. 1 Sample collection and general sample microbiome composition. a The general study design is

883 outlined describing the patient cohorts, per hospital feeding protocol, and prospective fecal sampling.

884 Infants born in two hospitals were enrolled shortly after birth and, per hospital protocol, assigned to a

885 feeding protocol based on gestational age at birth and weight at birth. Infants born at $>32$ weeks corrected

886 gestational age (cGA) and $1500 \mathrm{~g}$, were assigned to a feeding protocol which did not include a probiotic

887 (grey, top). Infants born at less than 32 weeks cGA and/or less than $1500 \mathrm{~g}$ were assigned to a feeding

888 protocol which included daily feedings of B. longum subsp. infantis EVC001 (8x109 CFU once per day)

889 starting with the initiation of trophic feeding (teal, bottom), and delivered in a single serving of $0.5 \mathrm{~mL}$

890 medium chain triglyceride oil. Nurses collected fecal samples (brown circles) opportunistically throughout

891 the patient's stay in the neonatal intensive care unit (NICU), aiming for two or more samples per week.

892 Infants received human milk (maternal or donor) through 34 weeks corrected gestational age, as well as

893 human milk-based human milk fortifier. After 34 weeks, infant formula was used in addition to human

894 milk. b Shotgun metagenome sequencing performed on samples collected from all 77 infants as used to

895 classify the average community structure of the infant microbiome in all samples. Individual sample 
compositions were generally similar and typified by either: $\mathbf{c}$ a high abundance of Bifidobacterium longum

897 species (inclusive of B. longum subsp. infantis; Bifidobacteriaceae, blue), d a high abundance of

898 Staphylococcus epidermidis (Staphylococcaceae, grey) and Enterobacteriaceae (red), or e an

899 overwhelming abundance of Escherichia coli and unclassified Escherichia (Enterobacteriaceae, red).

901 Fig. 2 Associations between gut taxa and clinical interventions. a Heatmap describing the output of 902 multivariate analysis of individual clinical variables (columns) with family-level microbial groups. This 903 approach separates the effects of individual clinical variables, and reports the impact of each one, 904 individually, on the abundance of the given bacterial family (row). Significant associations are indicated 905 by the notation of the FDR-adjusted $P$ value (asterisks), and the degree of the interaction (coefficient) is 906 indicated by the color scale. Strong, positive associations between a given clinical variable (row) and the 907 bacterial family (columns) are indicated by yellow, while strong negative associations between a clinical 908 variable and a bacterial family are indicated by dark blue. Weak associations (i.e. close to 0) are indicated 909 by green. Only bacterial families that included a significant association prior to FDR-correction are shown. 910 As Bifidobacteriaceae are strongly linked to probiotic intervention (as indicated by the yellow tile), we 911 examined interactions between this family and its constituents and other bacterial groups among infants fed 912 B. infantis EVC001.b) At the family level, Bifidobacteriaceae are strongly negatively correlated with 913 Enterobacteriaceae, as assessed using relative abundance and Spearman's rho ( $\rho$ ), among infants fed $B$. 914 infantis EVC001. Points are shaded based on the abundance of Bifidobacteriaceae. This is also true for two 915 of the primary constituents of the Enterobacteriaceae family; c) E. coli and B. longum species are negatively 916 correlated among infants fed B. infantis EVC001, and d B. longum is negatively correlated with $K$. 917 pneumoniae among infants fed $B$. infantis EVC001. (FDR-adjusted $P$ values are indicated by asterisks, $918 * * * *, P<0.0001 ; * * * ; P<0.001, * * ; P<0.01, * ; P<0.05)$

920 Fig. 3 Infant fecal samples collected over time reflect feeding protocols and improved development 921 trajectories with $B$. infantis EVC001. a Infant fecal samples (squares) collected over time, arranged by 
922 individual patient (y-axis) and corrected gestational age at sample collection, and ordered by gestational

923 age of the infant at birth (from earliest gestational age at birth, bottom, to oldest gestational age at birth,

924 top) and shaded by the abundance of Bifidobacteriaceae (see color scale). Infant feeding protocol is denoted

925 by the teal (EVC001-fed) or grey box (No Probiotic). Note that the feeding protocol assignment is based

926 both on gestational age at birth and birth weight, so some subjects (e.g. 302, 335) were assigned to the

927 probiotic feeding protocol based on weight at birth (i.e. $<1500$ g). b Fecal sample composition was

928 clustered by species-level signature taxa (e.g. B longum species, Escherichia coli and unclassified

929 Escherichia, and S. epidermidis), as indicated by color. Infants in the EVC001-fed feeding protocol more

930 frequently developed a gut microbiome composed of primarily $B$. longum species (blue), while the infants

931 not assigned to receive the probiotic frequently had compositions typified by high levels of

932 Enterobacteriaceae (e.g. Escherichia species) and/or Staphylococcus (red and orange, respectively). c Over

933 time, infants fed B. infantis EVC001 developed a high mean abundance of Bifidobacteriaceae (blue line)

934 which increased as infants approached term gestational age, which and this was negatively associated with

935 the abundance of Enterobacteriaceae (red line). d In contrast, among infants not fed B. infantis EVC001,

936 the abundance of Enterobacteriaceae did not change as infants approached term gestational age.

938 Fig. 4 Human milk oligosaccharide (HMO) utilization in B. infantis and the enrichment of HMO-

939 utilizing functions among infants fed B. infantis EVC001. a Bifidobacterium longum subsp. infantis (B.

940 infantis) utilizes a suite of human milk oligosaccharide (HMO)-associated gene loci to transport intact

941 HMO structures into the cell, where intracellularly located glycosyl hydrolases (blue) break down linkages

942 found commonly among HMO structures. Other enzymes involved in central carbohydrate metabolism

943 (teal) are used to convert sugars to the primary metabolic outputs, acetate and lactate. Other features

944 associated with HMO utilization include regulatory elements (purple) and urea metabolism (orange). b

945 These functions, which are represented as distinct KEGG orthologs, are differentially abundant among

946 samples, depending on the predominant bacterial species. Samples (columns) with greater abundance (i.e.

947 higher CPM, counts per million) of HMO-associated KEGG orthology (rows) were found among the $B$. 
longum species cluster. c All six functional categories were significantly enriched (i.e. greater CPM) in

949 samples from the $B$. longum species group (blue boxplot), relative to samples composed primarily of $S$.

950 epidermidis (orange boxplot) or Escherichia spp (red boxplot). This difference is associated with probiotic

951 B. infantis EVC001 feeding $\mathbf{d}$ as these functional categories were enriched among samples from infants fed

952 EVC001, relative to samples from infants who were not. ( $P$ values, adjusted for multiple comparisons, are

953 indicated by asterisks, ****, $P<0.0001$; ***, $P<0.001$; **, $P<0.01$; *, $P<0.05)$. Image created with

954 BioRender.com.

Fig. 5 Antibiotic resistant bacteria and antibiotic resistance genes are reduced among infants fed $B$.

957 infantis EVC001, irrespective of hospital location. a The relative proportion of antibiotic resistance genes

958 identified among taxa in fecal metagenomes. Nearly all ARGs could be assigned to Enterobacteriaceae

959 (red), Enterococcaceae (yellow), or Staphylococcaceae (green), though some could not be confidently

960 assigned to one organism (purple). b Infants transferred between hospitals participating in this study

961 demonstrated rapid colonization by bacteria harboring hospital-specific ARGs after their transfer date

962 (circle, age in days at transfer). All four transferred infants were assigned to the feeding protocol including

963 B. infantis EVC001, and the amount of hospital-specific ARGs trended lower and similar to other infants

964 in the unit that received B. infantis EVC001 (teal line, confidence intervals), relative to infants who did not

965 receive the probiotic (grey line, confidence intervals). c Infants at the two hospitals developed similar

966 patterns of ARG colonization, with more than 100 different unique ARGs detected. Infants acquired ARGs

967 throughout their stay, though infants fed B. infantis EVC001 tended to acquire fewer unique ARGs (teal),

968 relative to infants who did not receive the probiotic (grey). d Samples from infants fed B. infantis EVC001

969 also exhibited a lower overall relative abundance of ARGs (teal), as a proportion of their microbiome,

970 compared to samples from infants who did not receive the probiotic (grey). ( $P$ values indicated by asterisks,

$971 * * * *, P<0.0001)$

972 


\section{Fig. 6 Proinflammatory biomarkers are significantly lower in preterm infants fed $B$. infantis EVC001.}

974 a Multivariate analysis by linear models (MaAsLin2) was used to assess the effect that each clinical variable

975 had, independently, on proinflammatory biomarker concentrations. Probiotic feeding (B. infantis EVC001)

976 was significantly associated with lower TNFa concentrations (-1.06 pg/mL per kg per day; FDR-corrected

$977 P$ value $=0.0011)$. TPN $(\mathrm{mL} / \mathrm{kg} *$ day $)$ correlated with increased IL-8 production $(1.25 \mathrm{pg} / \mathrm{mL}$ per unit

978 increase; FDR-corrected $P$ value $=0.02)$ and a decrease of IL- $1 \beta$ and TNF $\alpha(0.064$ and $0.1 \mathrm{pg} / \mathrm{mL} *$ day;

979 FDR-corrected $P$ value $=0.02,0.02$, respectively). Day of life at sampling associated with decreased

980 calprotectin, IL-1 $\beta, \mathrm{IL}-8$, and TNF $\alpha$ production at a rate of $0.04,0.07,0.1$, and $0.08 \mathrm{pg} / \mathrm{mL} *$ day,

981 respectively (FDR-corrected $P$ value $=0.0001,0.0001,0.002$, and 0.0001 , respectively). No significant

982 associations between inhaled steroid exposure, diaper rash and treatment, antibiotic exposure, formula

983 feeding, human milk consumption, birth weight, or gestational age at birth was identified. b Significantly

984 decreased proinflammatory cytokine, IL-8 correlated with $B$. infantis EVC001 absolute abundance $(P=$

$9850.001, \rho=-0.24)$. $\mathbf{c}$ The mean relative abundance of bacterial families in samples analyzed at the $\sim 34$ week

986 timepoint, colored by family, is shown. Mean and standard deviations are available in Table S5. d-i Box

987 plots represent fecal calprotectin and proinflammatory cytokine concentrations [pg/mg] from infants not

988 fed B. infantis EVC001 $(\mathrm{n}=28)$ and EVC001-fed infants $(\mathrm{n}=29)$ at 34 weeks corrected gestational age.

989 Calprotectin and cytokine concentrations were measured in duplicate using MesoScale Discoveries R and

990 U-plex, respectively. Statistical analysis was completed using Wilcoxon rank sum test. $P$-values were

991 adjusted using the FDR method and considered to be statistically significantly decreased if $* * * * P<$

$9920.0001 ; * * * P<0.001 ; * * P<0.01 ; P<0.05$.

993 


\section{Figures}

Figure 1
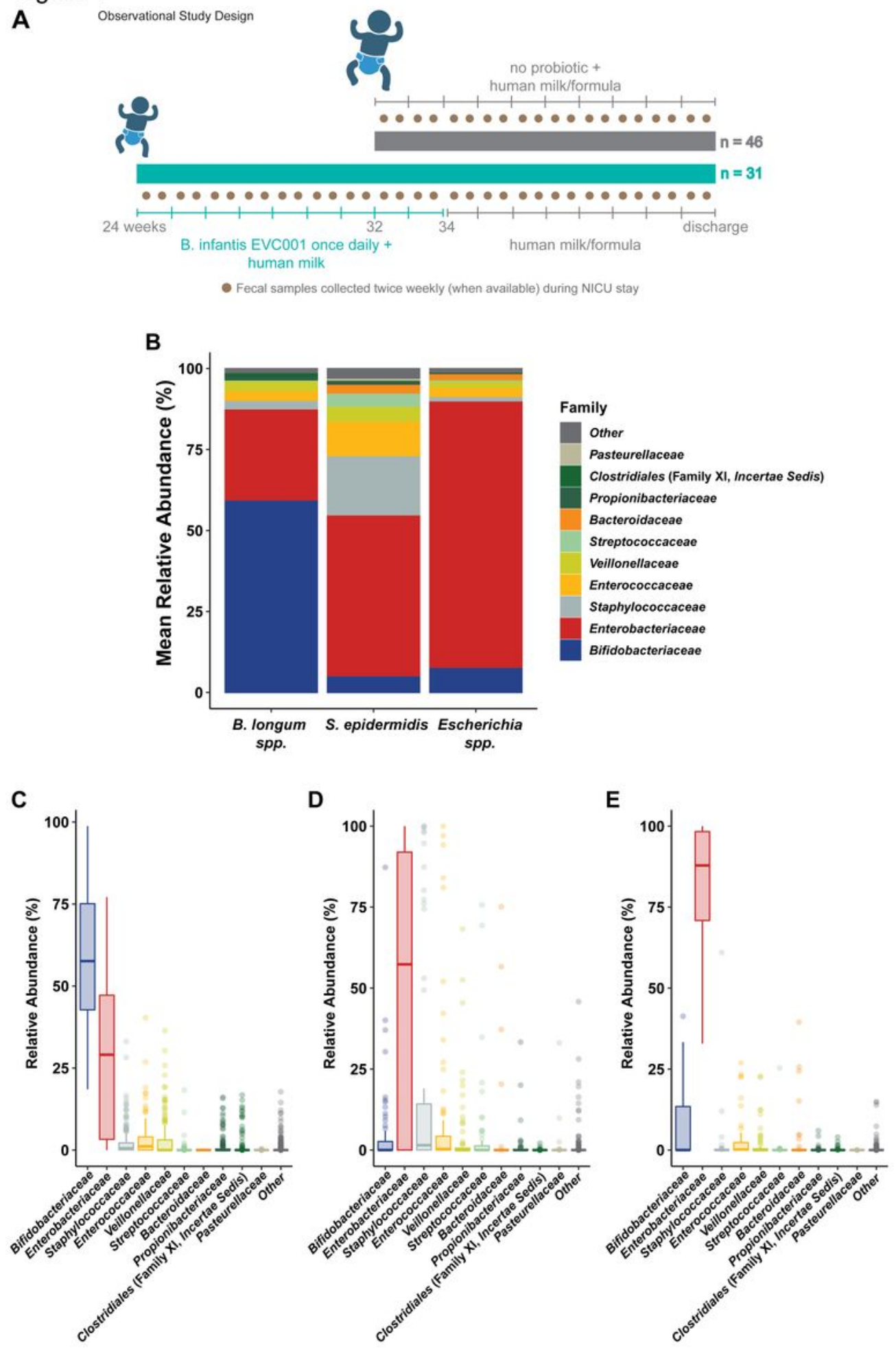

Figure 1

Sample collection and general sample microbiome composition. a The general study design is outlined describing the patient cohorts, per hospital feeding protocol, and prospective fecal sampling. Infants born in two hospitals were enrolled shortly after birth and, per hospital protocol, assigned to a feeding protocol 
based on gestational age at birth and weight at birth. Infants born at $>32$ weeks corrected gestational age (CGA) and $1500 \mathrm{~g}$, were assigned to a feeding protocol which did not include a probiotic (grey, top). Infants born at less than 32 weeks cGA and/or less than $1500 \mathrm{~g}$ were assigned to a feeding protocol which included daily feedings of B. longum subsp. infantis EVC001 (8x109 CFU once per day) starting with the initiation of trophic feeding (teal, bottom), and delivered in a single serving of $0.5 \mathrm{~mL}$ medium chain triglyceride oil. Nurses collected fecal samples (brown circles) opportunistically throughout the patient's stay in the neonatal intensive care unit (NICU), aiming for two or more samples per week. Infants received human milk (maternal or donor) through 34 weeks corrected gestational age, as well as human milk-based human milk fortifier. After 34 weeks, infant formula was used in addition to human milk. $b$ Shotgun metagenome sequencing performed on samples collected from all 77 infants as used to classify the average community structure of the infant microbiome in all samples. Individual sample compositions were generally similar and typified by either: $\mathrm{c}$ a high abundance of Bifidobacterium 896 longum species (inclusive of B. longum subsp. infantis; Bifidobacteriaceae, blue), $d$ a high abundance of Staphylococcus epidermidis (Staphylococcaceae, grey) and Enterobacteriaceae (red), or e an overwhelming abundance of Escherichia coli and unclassified Escherichia (Enterobacteriaceae, red). 
Figure 1

A

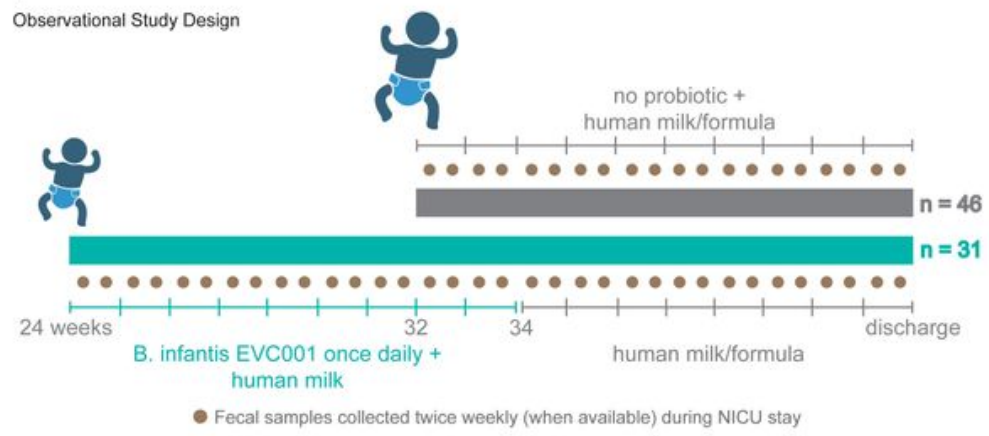

B
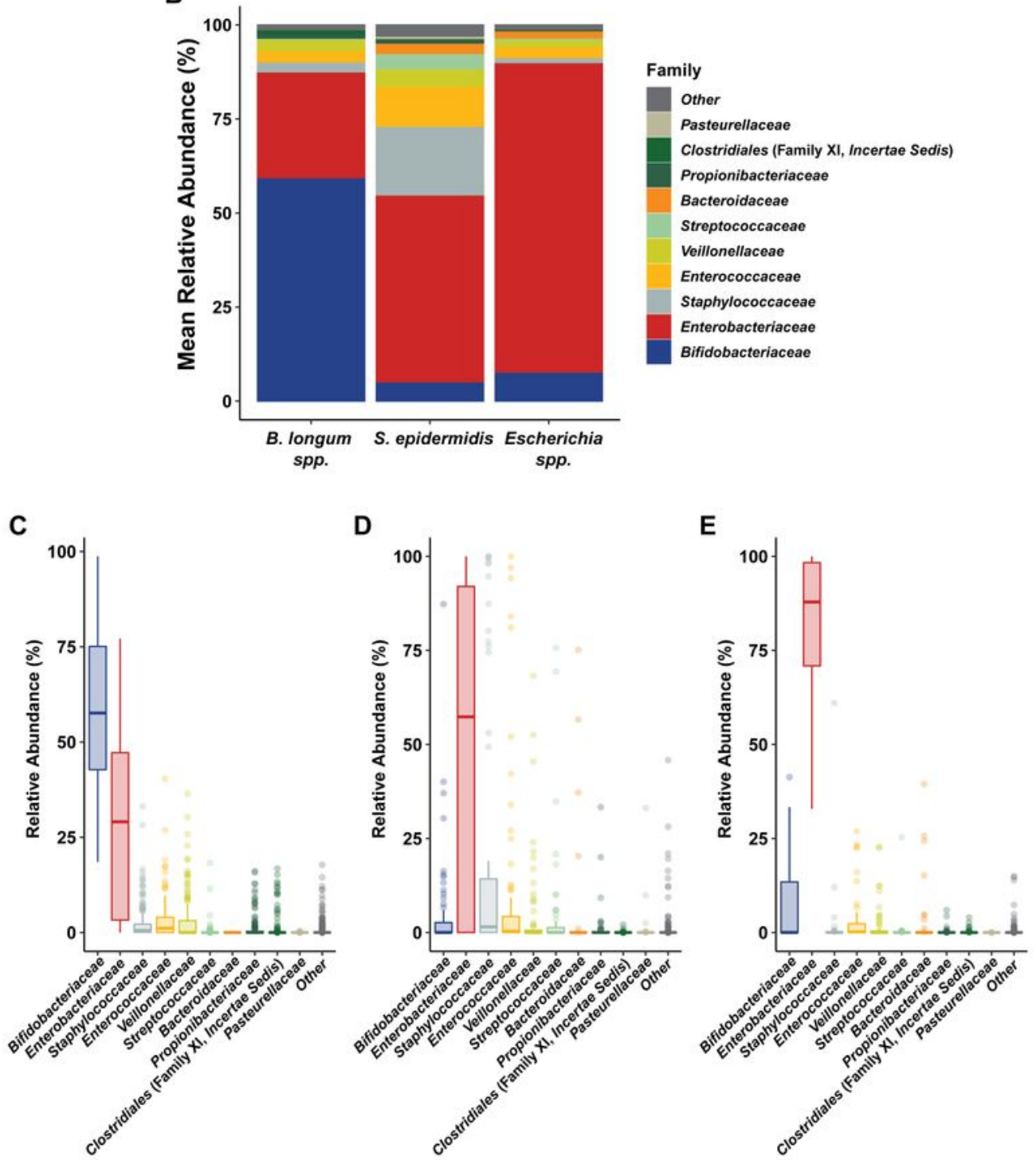

\section{Figure 1}

Sample collection and general sample microbiome composition. a The general study design is outlined describing the patient cohorts, per hospital feeding protocol, and prospective fecal sampling. Infants born in two hospitals were enrolled shortly after birth and, per hospital protocol, assigned to a feeding protocol based on gestational age at birth and weight at birth. Infants born at > 32 weeks corrected gestational age (cGA) and $1500 \mathrm{~g}$, were assigned to a feeding protocol which did not include a probiotic (grey, top). 
Infants born at less than 32 weeks cGA and/or less than $1500 \mathrm{~g}$ were assigned to a feeding protocol which included daily feedings of B. longum subsp. infantis EVC001 (8x109 CFU once per day) starting with the initiation of trophic feeding (teal, bottom), and delivered in a single serving of $0.5 \mathrm{~mL}$ medium chain triglyceride oil. Nurses collected fecal samples (brown circles) opportunistically throughout the patient's stay in the neonatal intensive care unit (NICU), aiming for two or more samples per week. Infants received human milk (maternal or donor) through 34 weeks corrected gestational age, as well as human milk-based human milk fortifier. After 34 weeks, infant formula was used in addition to human milk. b Shotgun metagenome sequencing performed on samples collected from all 77 infants as used to classify the average community structure of the infant microbiome in all samples. Individual sample compositions were generally similar and typified by either: $\mathrm{c}$ a high abundance of Bifidobacterium 896 longum species (inclusive of B. longum subsp. infantis; Bifidobacteriaceae, blue), $d$ a high abundance of Staphylococcus epidermidis (Staphylococcaceae, grey) and Enterobacteriaceae (red), or e an overwhelming abundance of Escherichia coli and unclassified Escherichia (Enterobacteriaceae, red). 


\section{A}

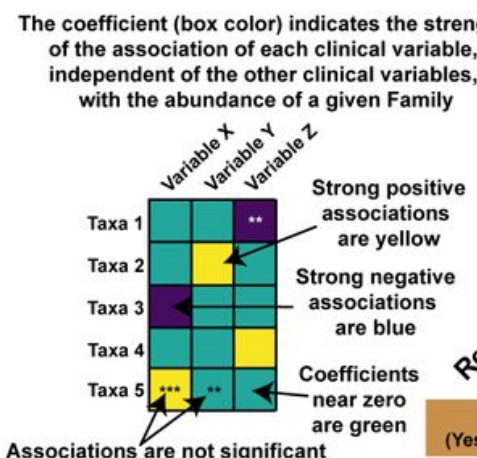

Associations are not significant unless a $P$ value is indicated

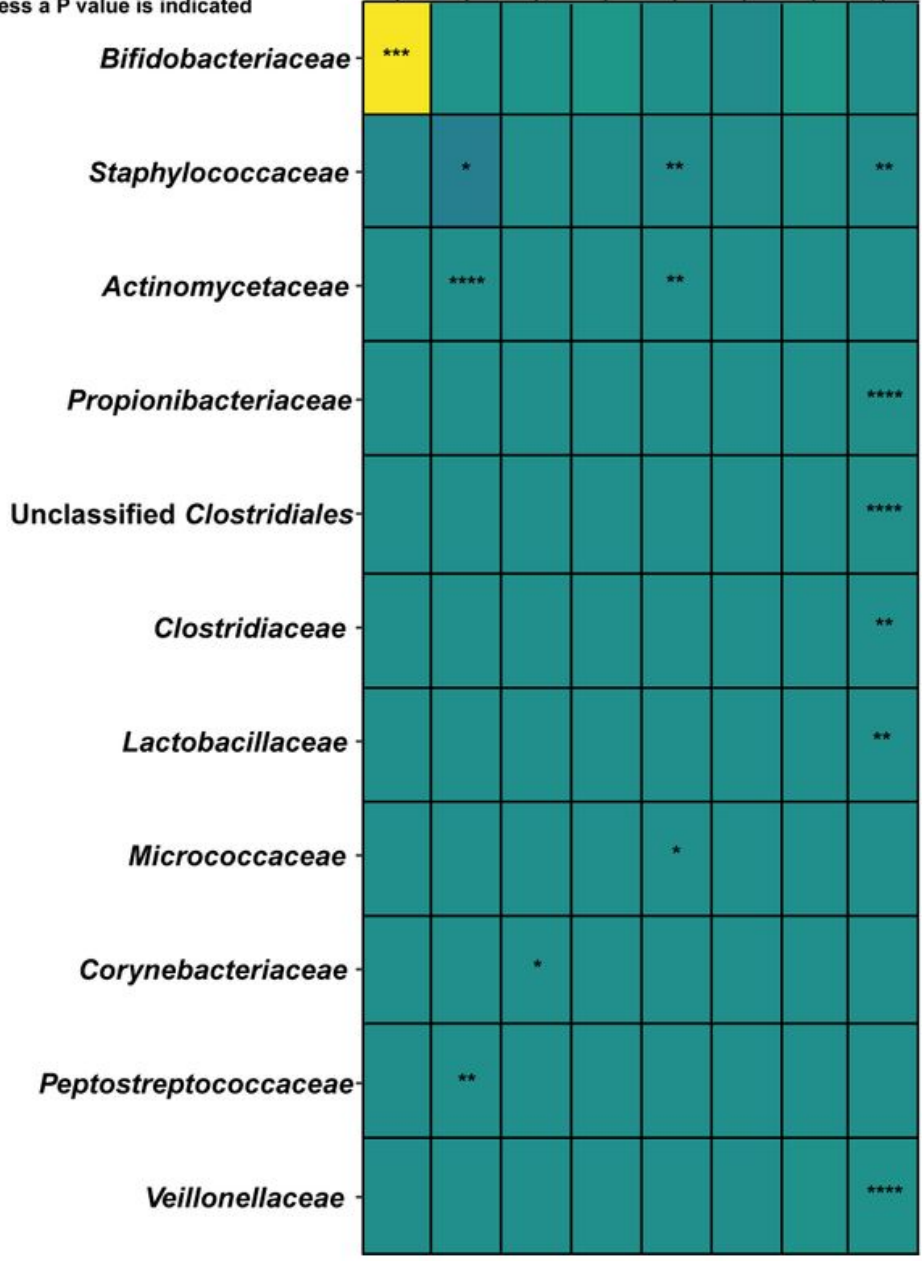

B

Coefficient

Bifidobacteriaceae (\%)

$-20-100 \quad 1020$

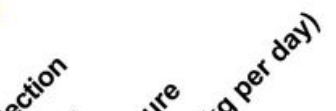

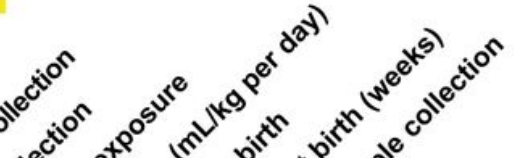

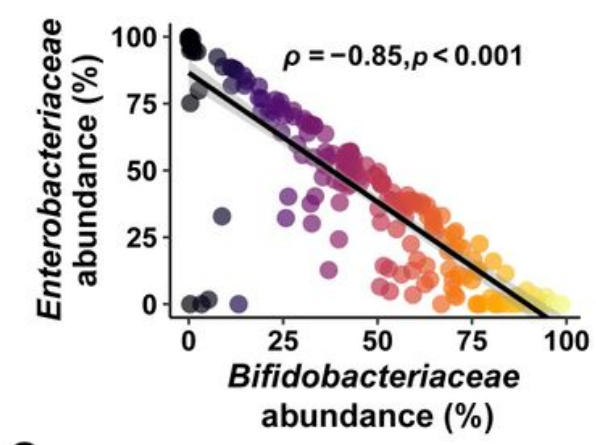

C
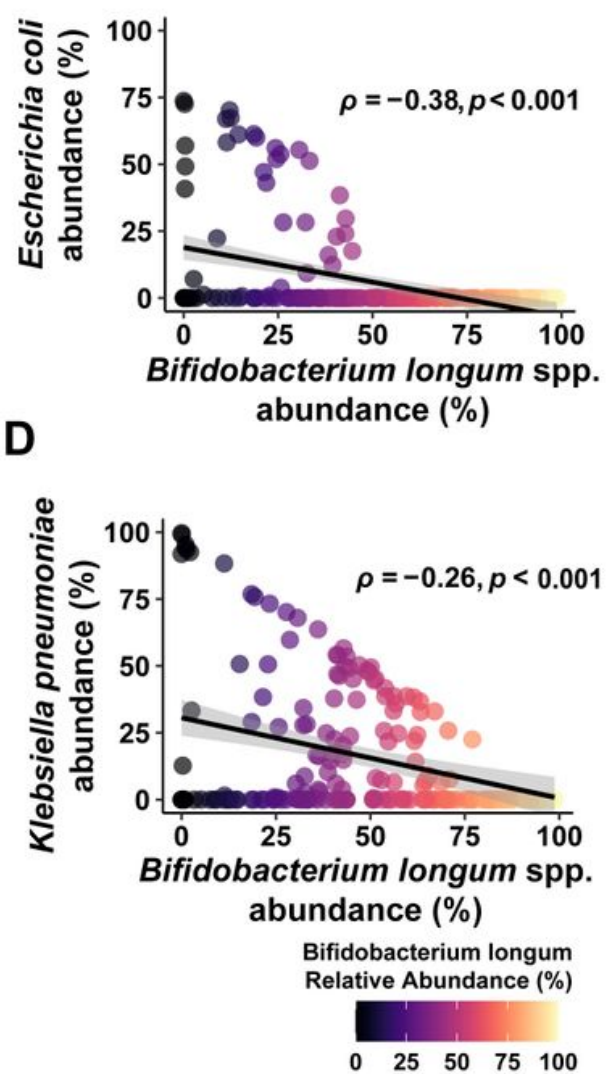

Figure 2

Associations between gut taxa and clinical interventions. a Heatmap describing the output of multivariate analysis of individual clinical variables (columns) with family-level microbial groups. This approach separates the effects of individual clinical variables, and reports the impact of each one, individually, on the abundance of the given bacterial family (row). Significant associations are indicated by the notation of the FDR-adjusted P value (asterisks), and the degree of the interaction (coefficient) is indicated by the color scale. Strong, positive associations between a given clinical variable (row) and the bacterial family (columns) are indicated by yellow, while strong negative associations between a clinical variable and a 
bacterial family are indicated by dark blue. Weak associations (i.e. close to 0 ) are indicated by green. Only bacterial families that included a significant association prior to FDR-correction are shown. As Bifidobacteriaceae are strongly linked to probiotic intervention (as indicated by the yellow tile), we examined interactions between this family and its constituents and other bacterial groups among infants fed B. infantis EVC001.b) At the family level, Bifidobacteriaceae are strongly negatively correlated with Enterobacteriaceae, as assessed using relative abundance and Spearman's rho ( $\rho)$, among infants fed B. infantis EVC001. Points are shaded based on the abundance of Bifidobacteriaceae. This is also true for two of the primary constituents of the Enterobacteriaceae family; C) E. coli and B. Iongum species are negatively correlated among infants fed $\mathrm{B}$. infantis EVC001, and d B. longum is negatively correlated with K. pneumoniae among infants fed $B$. infantis EVC001. (FDR-adjusted $P$ values are indicated by asterisks, $\star \star \star \star, ~ P<0.0001 ; * \star \star ; P<0.001$, **; $P<0.01, * ; P<0.05)$ 


\section{A}

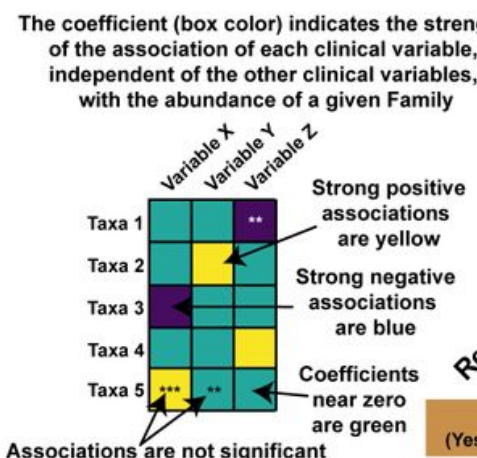

Associations are not significant unless a $P$ value is indicated

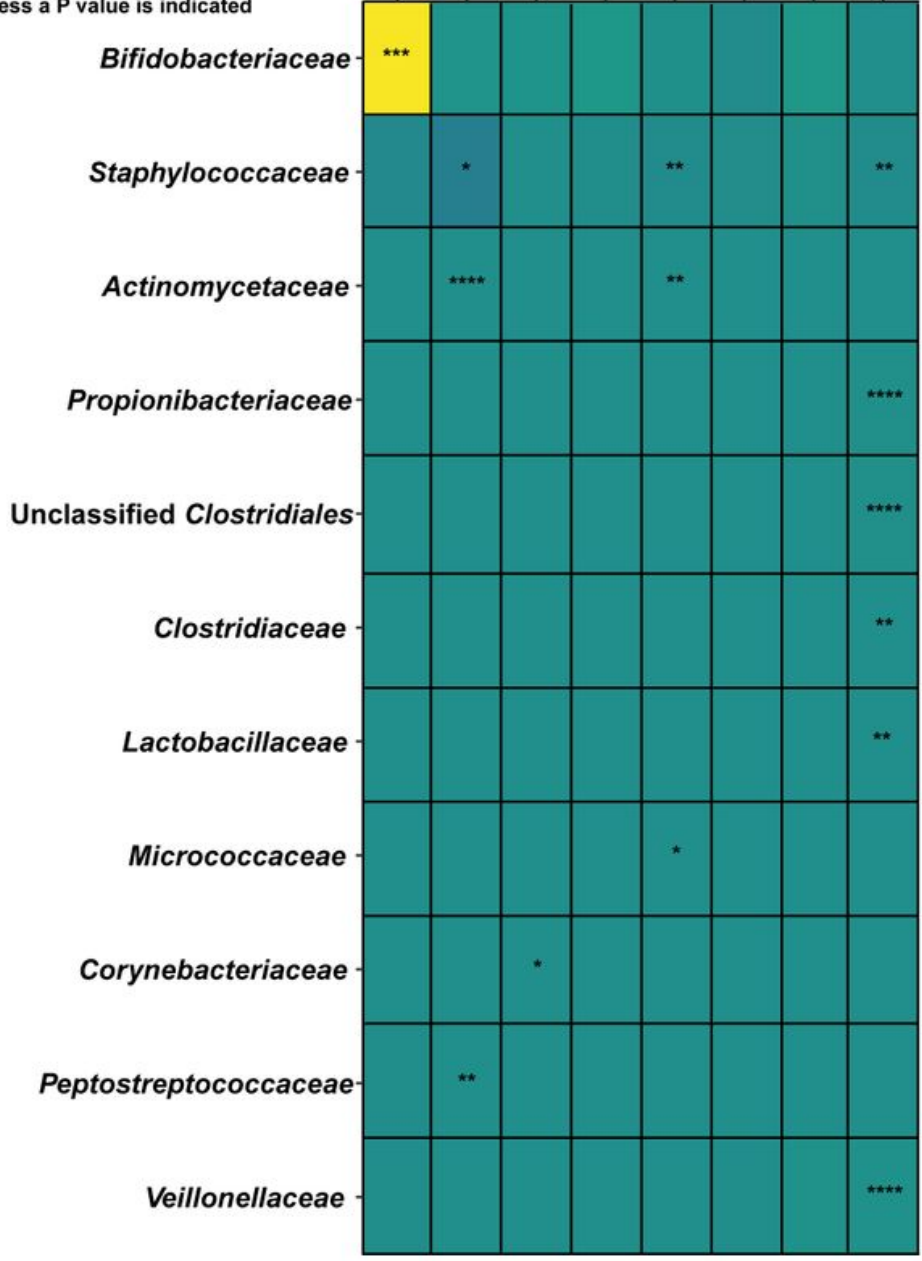

B

Coefficient

Bifidobacteriaceae (\%)

$-20-100 \quad 1020$

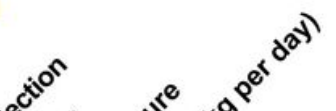

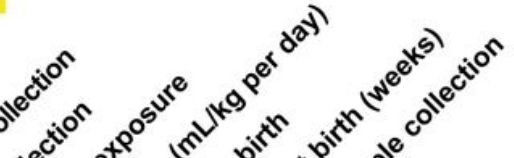

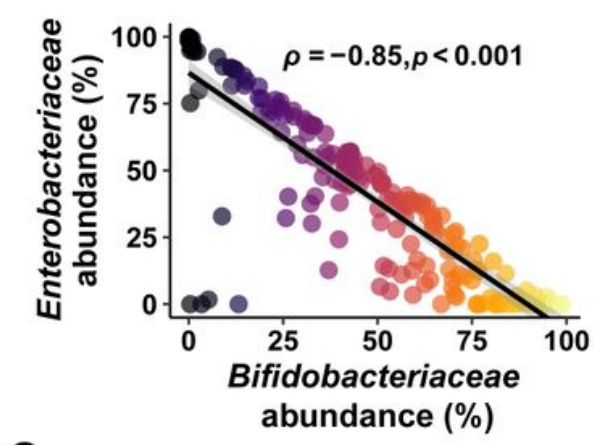

C
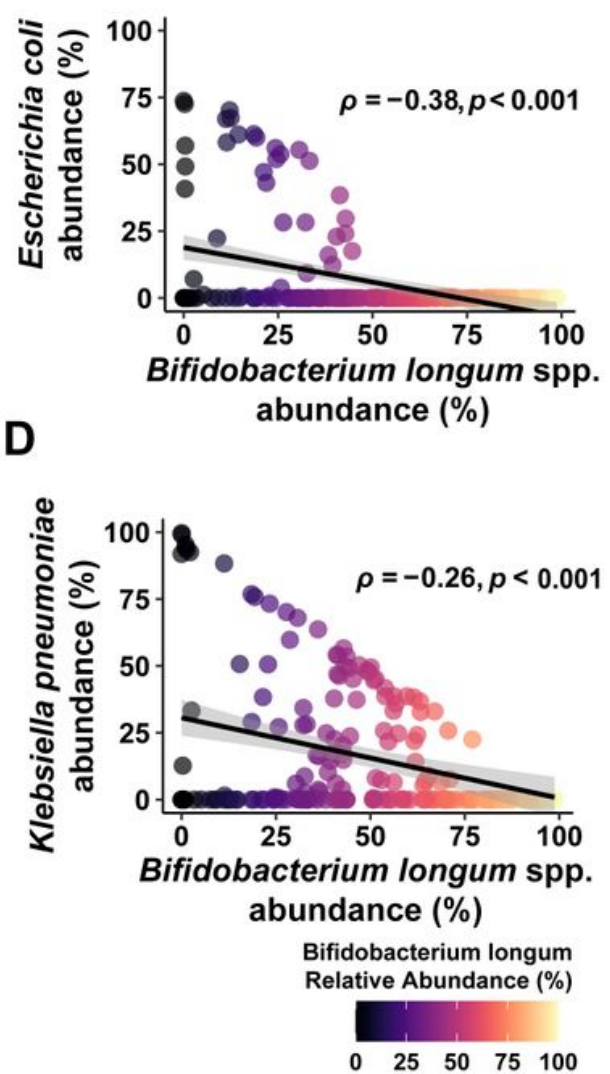

Figure 2

Associations between gut taxa and clinical interventions. a Heatmap describing the output of multivariate analysis of individual clinical variables (columns) with family-level microbial groups. This approach separates the effects of individual clinical variables, and reports the impact of each one, individually, on the abundance of the given bacterial family (row). Significant associations are indicated by the notation of the FDR-adjusted P value (asterisks), and the degree of the interaction (coefficient) is indicated by the color scale. Strong, positive associations between a given clinical variable (row) and the bacterial family (columns) are indicated by yellow, while strong negative associations between a clinical variable and a 
bacterial family are indicated by dark blue. Weak associations (i.e. close to 0 ) are indicated by green. Only bacterial families that included a significant association prior to FDR-correction are shown. As Bifidobacteriaceae are strongly linked to probiotic intervention (as indicated by the yellow tile), we examined interactions between this family and its constituents and other bacterial groups among infants fed B. infantis EVC001.b) At the family level, Bifidobacteriaceae are strongly negatively correlated with Enterobacteriaceae, as assessed using relative abundance and Spearman's rho ( $\rho)$, among infants fed B. infantis EVC001. Points are shaded based on the abundance of Bifidobacteriaceae. This is also true for two of the primary constituents of the Enterobacteriaceae family; C) E. coli and B. Iongum species are negatively correlated among infants fed $\mathrm{B}$. infantis EVC001, and d B. longum is negatively correlated with K. pneumoniae among infants fed $B$. infantis EVC001. (FDR-adjusted $P$ values are indicated by asterisks, $\star \star * \star, P<0.0001 ; * * * ; P<0.001$, **; $\mathrm{P}<0.01, * ; \mathrm{P}<0.05)$

\section{Figure 3}

A

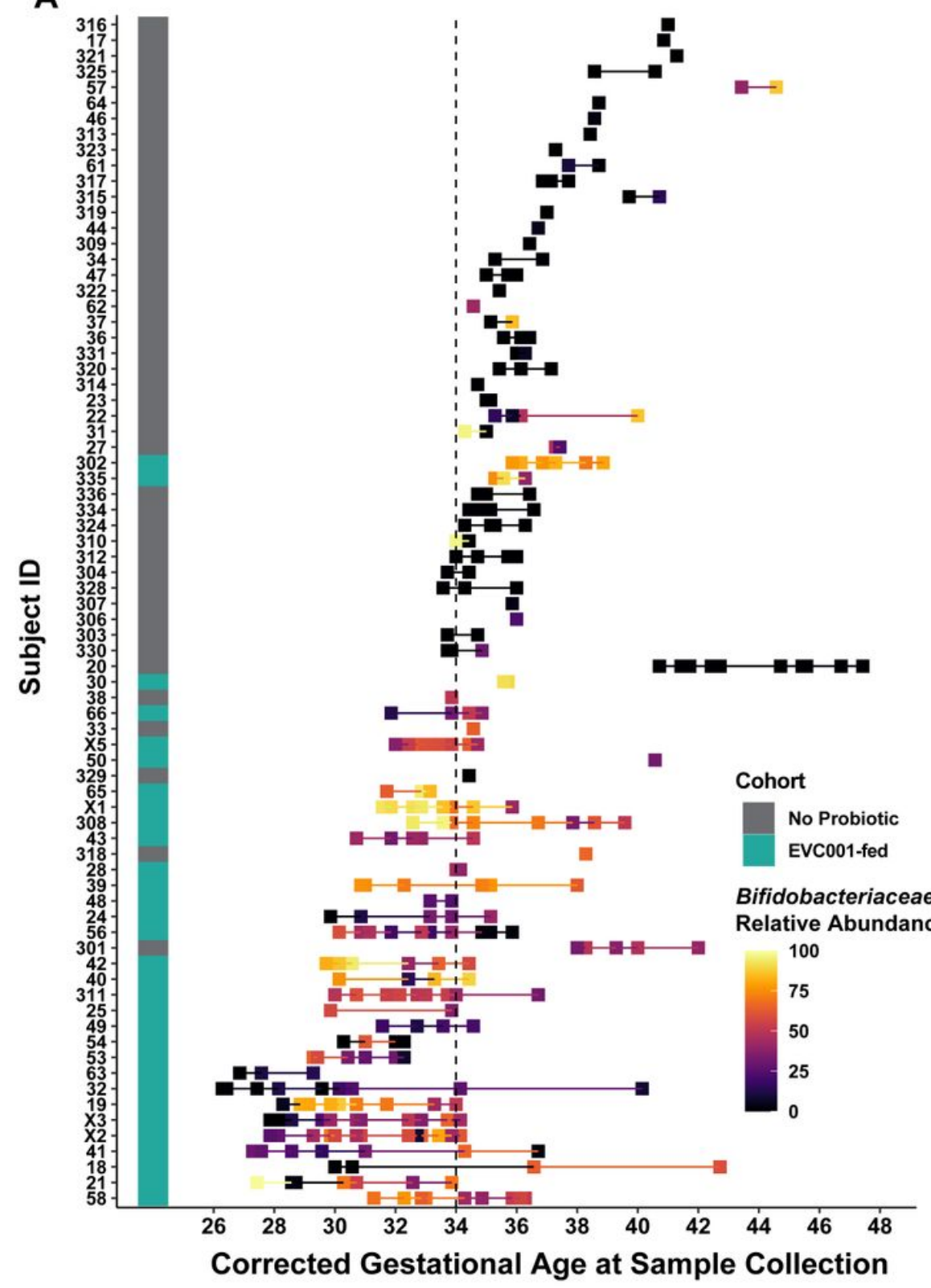

B

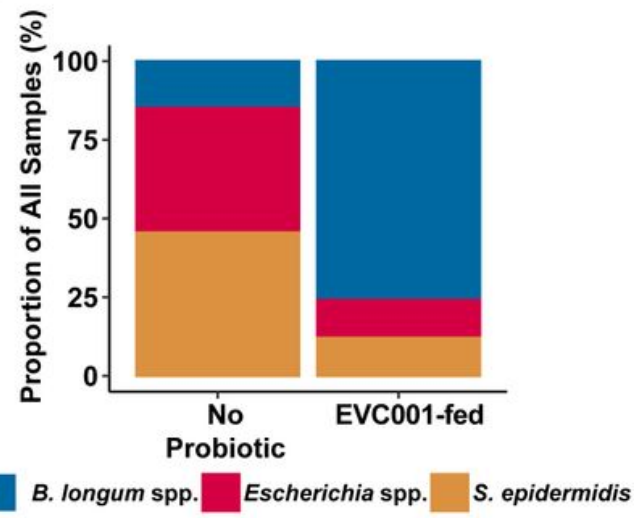

C

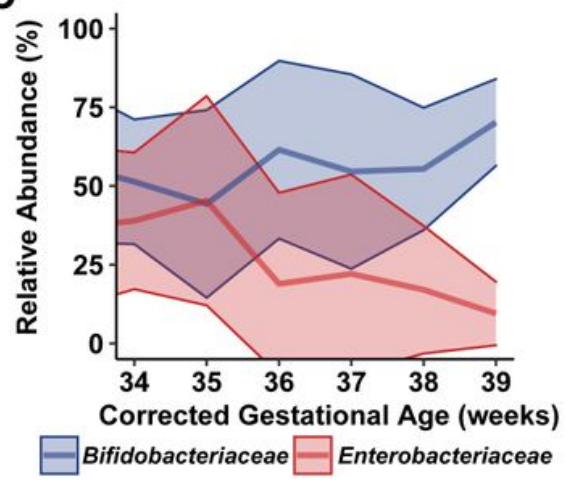

D

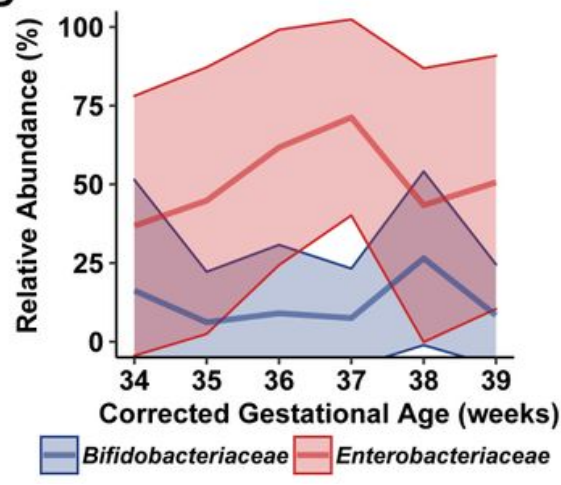

Figure 3 
Infant fecal samples collected over time reflect feeding protocols and improved development trajectories with B. infantis EVC001. a Infant fecal samples (squares) collected over time, arranged by individual patient (y-axis) and corrected gestational age at sample collection, and ordered by gestational age of the infant at birth (from earliest gestational age at birth, bottom, to oldest gestational age at birth, top) and shaded by the abundance of Bifidobacteriaceae (see color scale). Infant feeding protocol is denoted by the teal (EVC001-fed) or grey box (No Probiotic). Note that the feeding protocol assignment is based both on gestational age at birth and birth weight, so some subjects (e.g. 302, 335) were assigned to the probiotic feeding protocol based on weight at birth (i.e. $<1500 \mathrm{~g}$ ). b Fecal sample composition was clustered by species-level signature taxa (e.g. B longum species, Escherichia coli and unclassified Escherichia, and S. epidermidis), as indicated by color. Infants in the EVC001-fed feeding protocol more frequently developed a gut microbiome composed of primarily $B$. longum species (blue), while the infants not assigned to receive the probiotic frequently had compositions typified by high levels of Enterobacteriaceae (e.g. Escherichia species) and/or Staphylococcus (red and orange, respectively). c Over time, infants fed B. infantis EVC001 developed a high mean abundance of Bifidobacteriaceae (blue line) which increased as infants approached term gestational age, which and this was negatively associated with the abundance of Enterobacteriaceae (red line). $d$ In contrast, among infants not fed B. infantis EVC001, the abundance of Enterobacteriaceae did not change as infants approached term gestational age. 
Figure 3

A

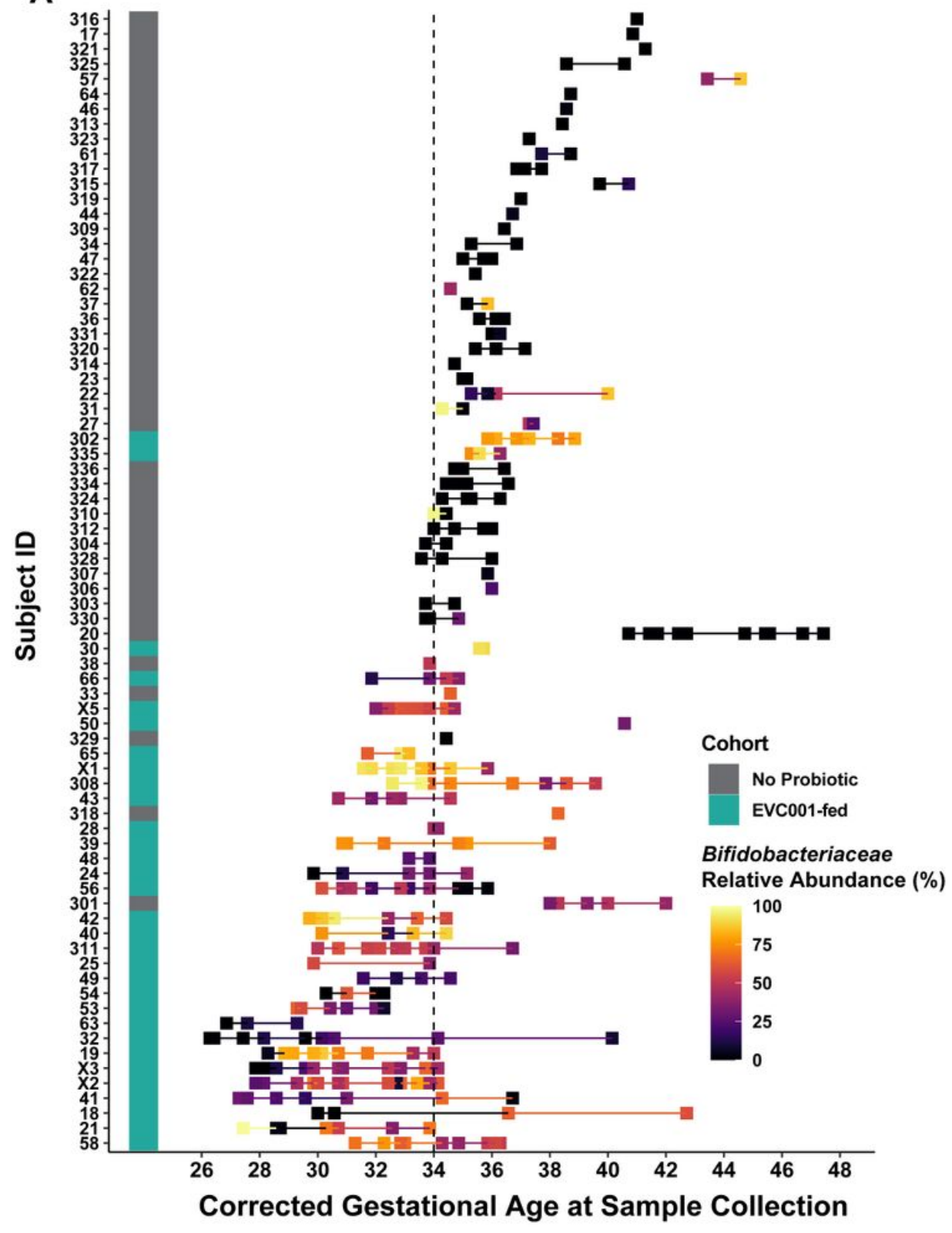

B

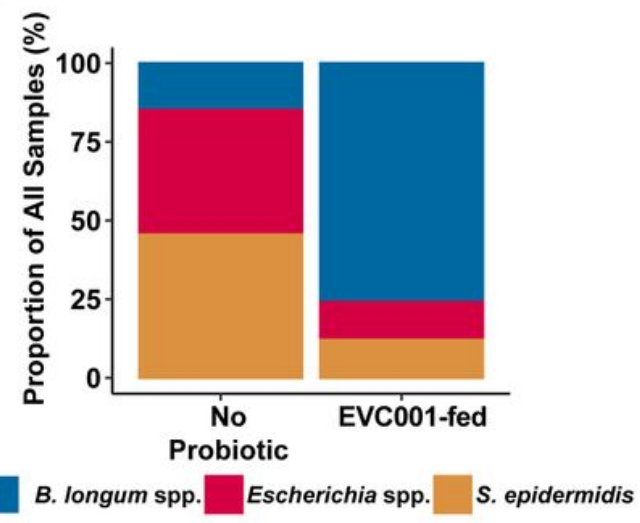

C

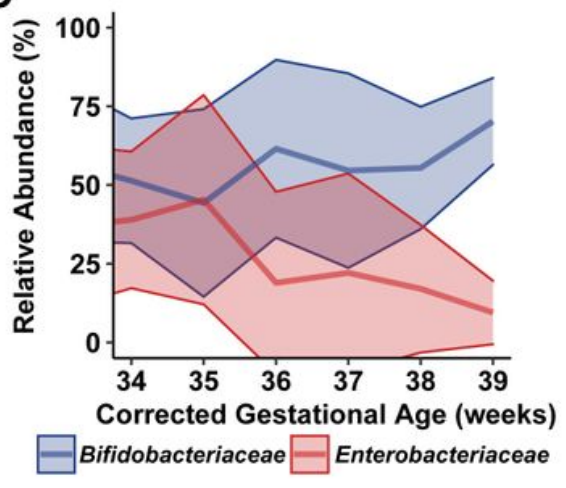

D

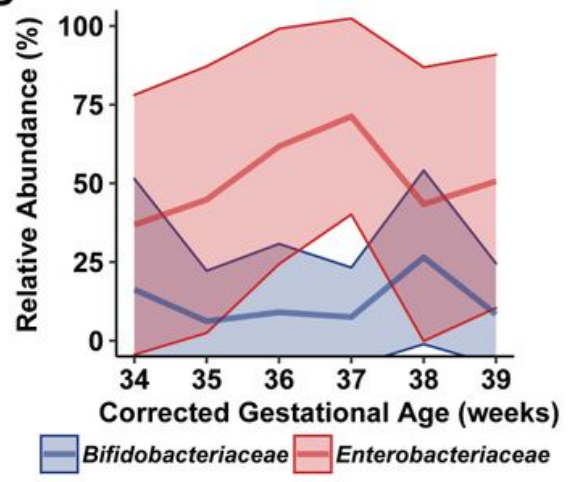

Figure 3

Infant fecal samples collected over time reflect feeding protocols and improved development trajectories with B. infantis EVC001. a Infant fecal samples (squares) collected over time, arranged by individual patient (y-axis) and corrected gestational age at sample collection, and ordered by gestational age of the infant at birth (from earliest gestational age at birth, bottom, to oldest gestational age at birth, top) and shaded by the abundance of Bifidobacteriaceae (see color scale). Infant feeding protocol is denoted by the teal (EVC001-fed) or grey box (No Probiotic). Note that the feeding protocol assignment is based both on gestational age at birth and birth weight, so some subjects (e.g. 302, 335) were assigned to the probiotic feeding protocol based on weight at birth (i.e. $<1500 \mathrm{~g}$ ). b Fecal sample composition was clustered by species-level signature taxa (e.g. B longum species, Escherichia coli and unclassified Escherichia, and S. epidermidis), as indicated by color. Infants in the EVC001-fed feeding protocol more 
frequently developed a gut microbiome composed of primarily B. longum species (blue), while the infants not assigned to receive the probiotic frequently had compositions typified by high levels of Enterobacteriaceae (e.g. Escherichia species) and/or Staphylococcus (red and orange, respectively). c Over time, infants fed B. infantis EVC001 developed a high mean abundance of Bifidobacteriaceae (blue line) which increased as infants approached term gestational age, which and this was negatively associated with the abundance of Enterobacteriaceae (red line). $d$ In contrast, among infants not fed B. infantis EVC001, the abundance of Enterobacteriaceae did not change as infants approached term gestational age. 

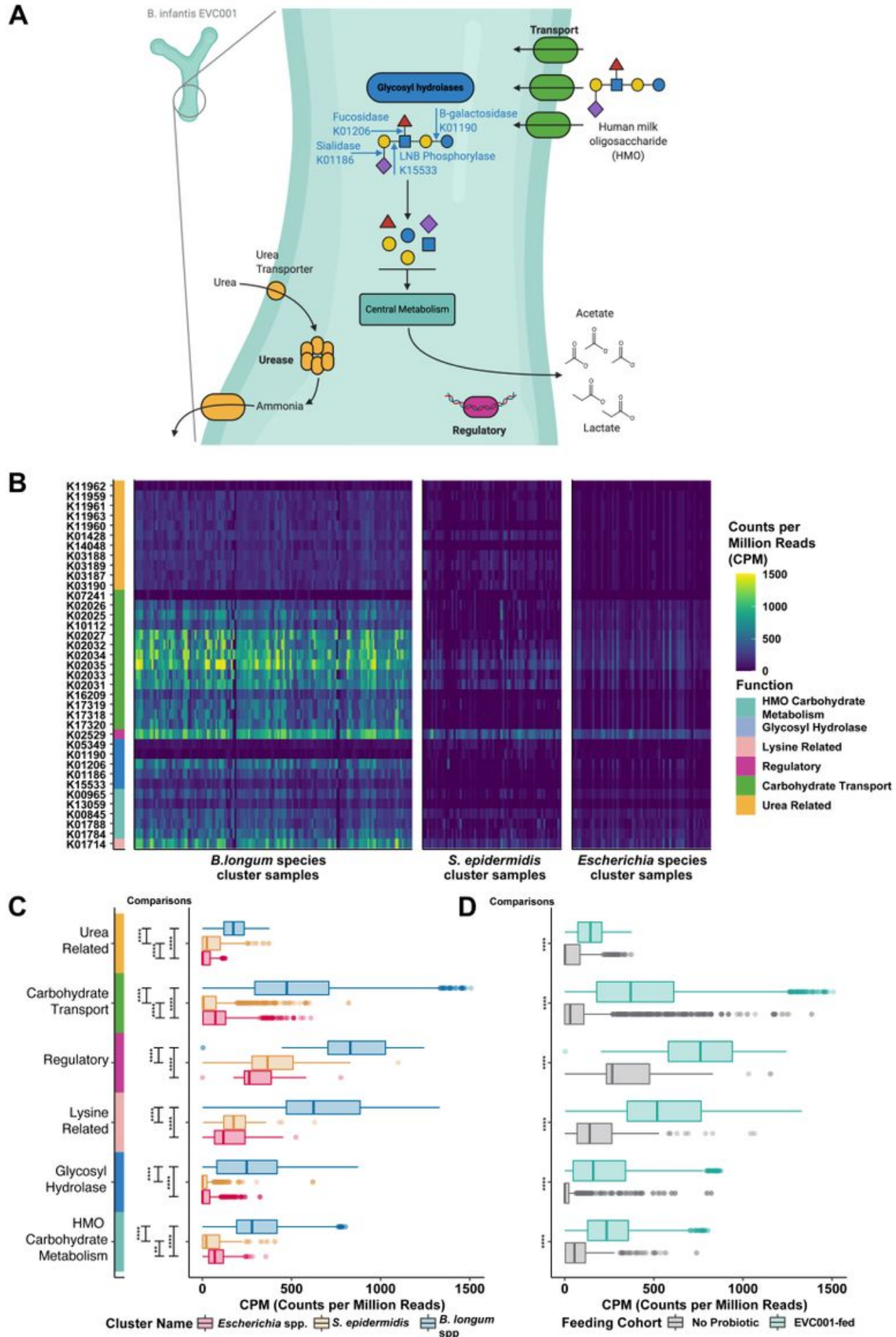

\section{Figure 4}

Human milk oligosaccharide (HMO) utilization in B. infantis and the enrichment of HMO939 utilizing functions among infants fed B. infantis EVC001. a Bifidobacterium longum subsp. infantis (B. infantis) utilizes a suite of human milk oligosaccharide (HMO)-associated gene loci to transport intact HMO structures into the cell, where intracellularly located glycosyl hydrolases (blue) break down linkages found commonly among HMO structures. Other enzymes involved in central carbohydrate metabolism (teal) are 
used to convert sugars to the primary metabolic outputs, acetate and lactate. Other features associated with $\mathrm{HMO}$ utilization include regulatory elements (purple) and urea metabolism (orange). b These functions, which are represented as distinct KEGG orthologs, are differentially abundant among samples, depending on the predominant bacterial species. Samples (columns) with greater abundance (i.e. higher CPM, counts per million) of HMO-associated KEGG orthology (rows) were found among the B. longum species cluster. c All six functional categories were significantly enriched (948 i.e. greater CPM) in samples from the B. longum species group (blue boxplot), relative to samples composed primarily of $S$. epidermidis (orange boxplot) or Escherichia spp (red boxplot). This difference is associated with probiotic B. infantis EVC001 feeding $d$ as these functional categories were enriched among samples from infants fed EVC001, relative to samples from infants who were not. ( $P$ values, adjusted for multiple comparisons,

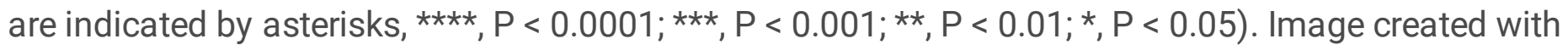
BioRender.com. 

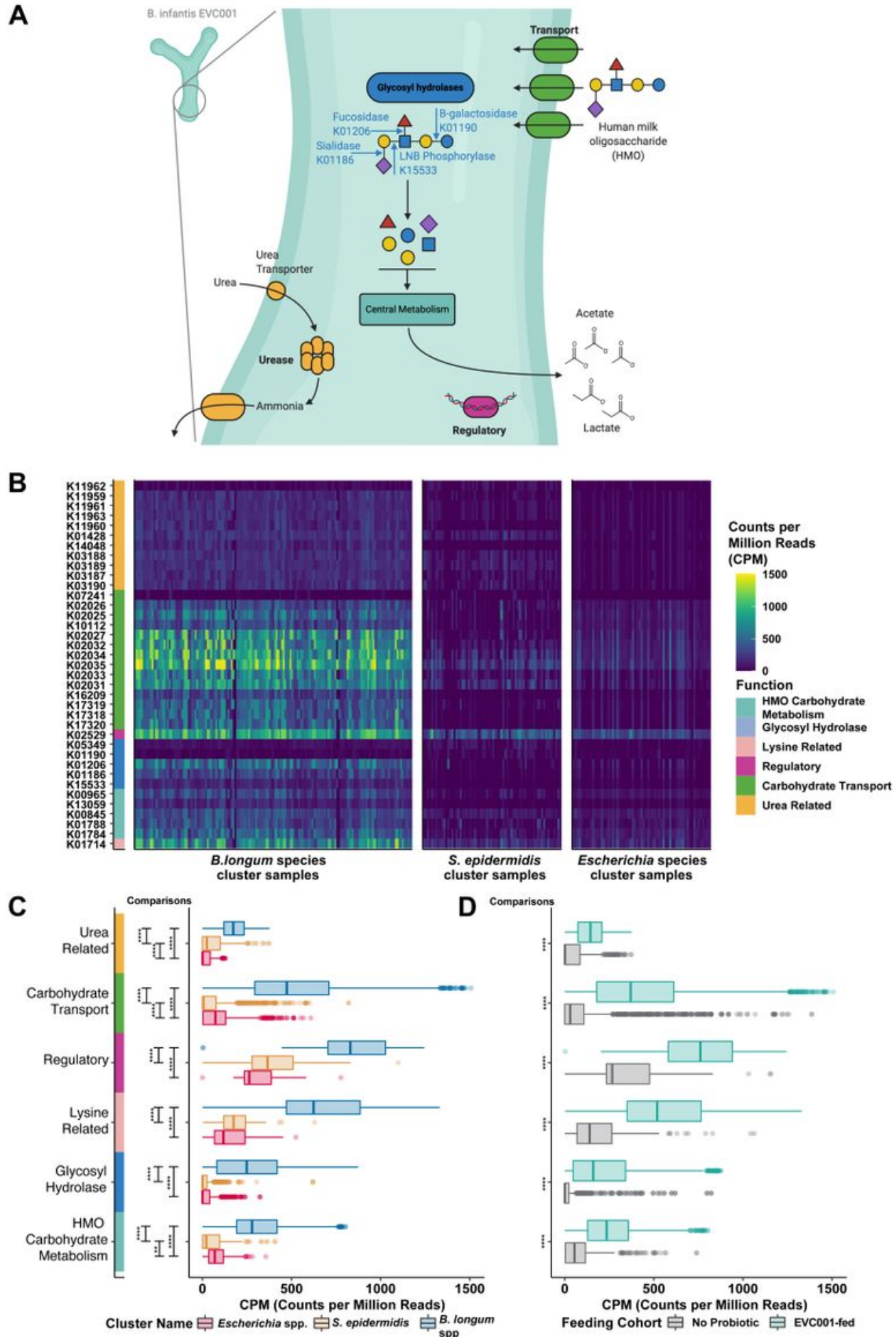

\section{Figure 4}

Human milk oligosaccharide (HMO) utilization in B. infantis and the enrichment of HMO939 utilizing functions among infants fed B. infantis EVC001. a Bifidobacterium longum subsp. infantis (B. infantis) utilizes a suite of human milk oligosaccharide (HMO)-associated gene loci to transport intact HMO structures into the cell, where intracellularly located glycosyl hydrolases (blue) break down linkages found commonly among HMO structures. Other enzymes involved in central carbohydrate metabolism (teal) are 
used to convert sugars to the primary metabolic outputs, acetate and lactate. Other features associated with $\mathrm{HMO}$ utilization include regulatory elements (purple) and urea metabolism (orange). b These functions, which are represented as distinct KEGG orthologs, are differentially abundant among samples, depending on the predominant bacterial species. Samples (columns) with greater abundance (i.e. higher $\mathrm{CPM}$, counts per million) of HMO-associated KEGG orthology (rows) were found among the B. longum species cluster. c All six functional categories were significantly enriched (948 i.e. greater CPM) in samples from the B. longum species group (blue boxplot), relative to samples composed primarily of $\mathrm{S}$. epidermidis (orange boxplot) or Escherichia spp (red boxplot). This difference is associated with probiotic $B$. infantis EVC001 feeding $d$ as these functional categories were enriched among samples from infants fed EVC001, relative to samples from infants who were not. ( $P$ values, adjusted for multiple comparisons,

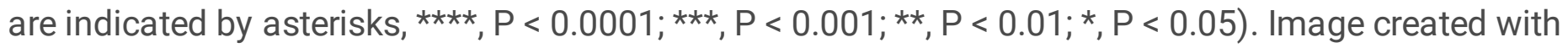
BioRender.com.

\section{Figure 5}
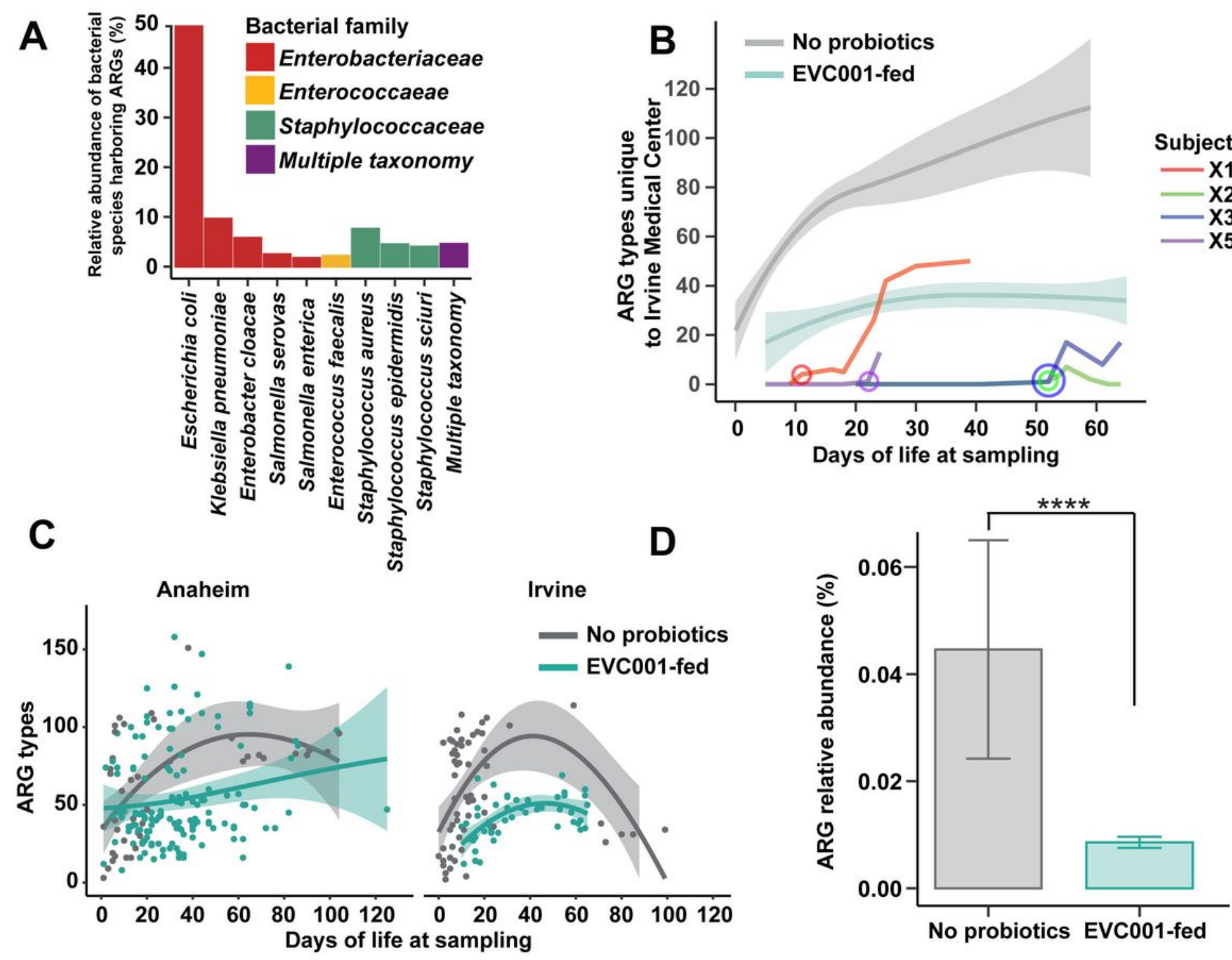

Figure 5 
Antibiotic resistant bacteria and antibiotic resistance genes are reduced among infants fed $B$. infantis EVC001, irrespective of hospital location. a The relative proportion of antibiotic resistance genes identified among taxa in fecal metagenomes. Nearly all ARGs could be assigned to Enterobacteriaceae (red), Enterococcaceae (yellow), or Staphylococcaceae (green), though some could not be confidently assigned to one organism (purple). b Infants transferred between hospitals participating in this study demonstrated rapid colonization by bacteria harboring hospital-specific ARGs after their transfer date (circle, age in days at transfer). All four transferred infants were assigned to the feeding protocol including B. infantis EVC001, and the amount of hospital-specific ARGs trended lower and similar to other infants in the unit that received B. infantis EVC001 (teal line, confidence intervals), relative to infants who did not receive the probiotic (grey line, confidence intervals). c Infants at the two hospitals developed similar patterns of ARG colonization, with more than 100 different unique ARGs detected. Infants acquired ARGs throughout their stay, though infants fed B. infantis EVC001 tended to acquire fewer unique ARGs (teal), relative to infants who did not receive the probiotic (grey). d Samples from infants fed B. infantis EVC001 also exhibited a lower overall relative abundance of ARGs (teal), as a proportion of their microbiome, compared to samples from infants who did not receive the probiotic (grey). ( $P$ values indicated by asterisks, $\star \star \star \star, ~ P<0.0001)$ 


\section{Figure 5}
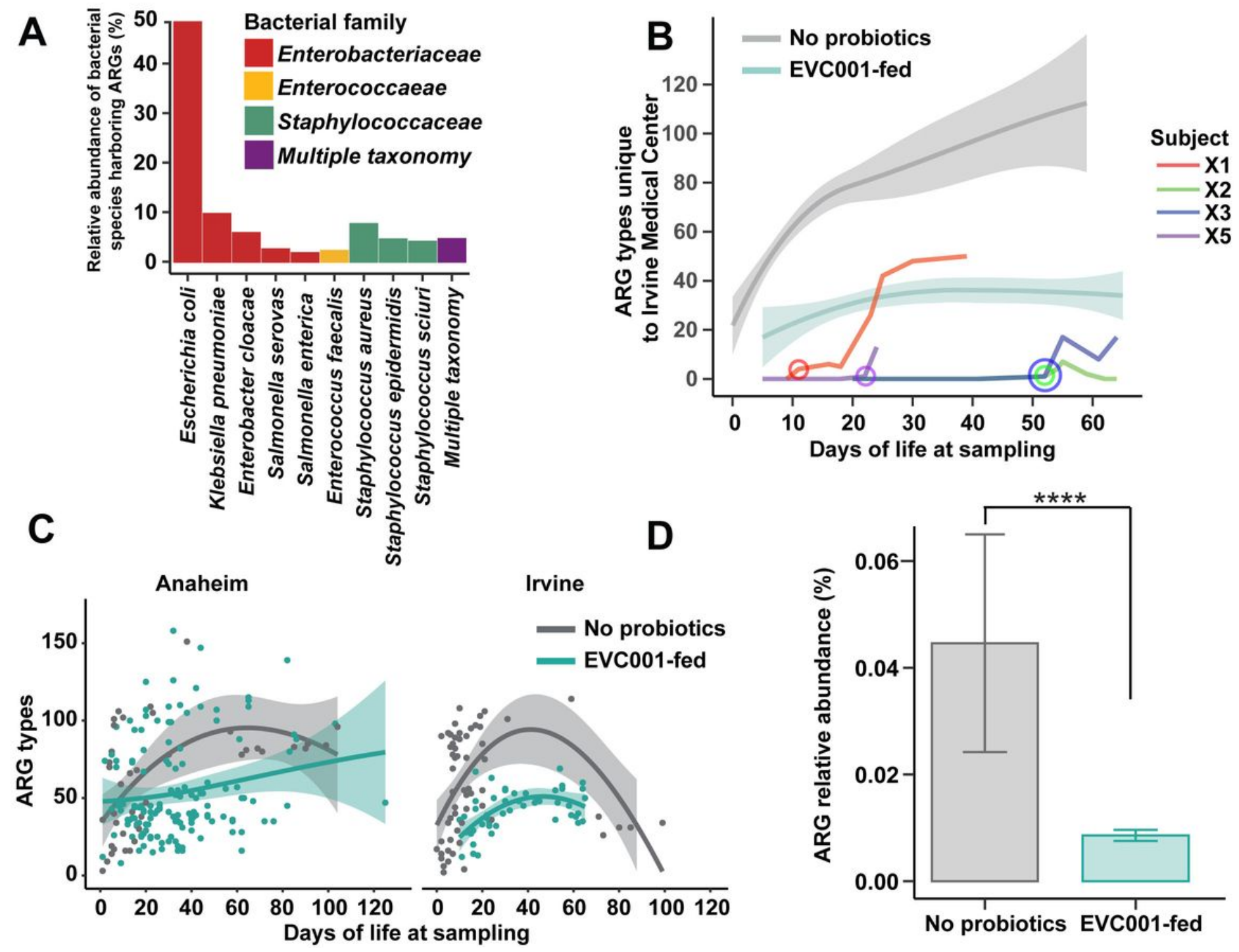

Figure 5

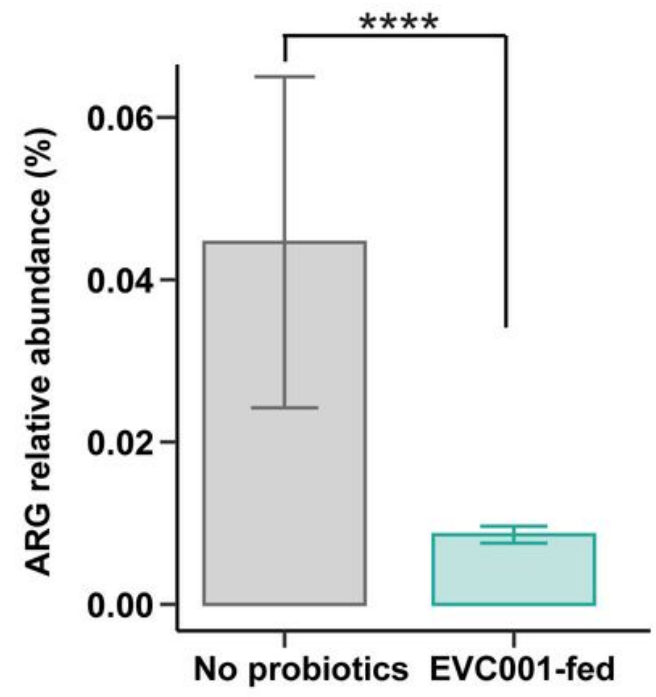

Antibiotic resistant bacteria and antibiotic resistance genes are reduced among infants fed $\mathrm{B}$. infantis EVC001, irrespective of hospital location. a The relative proportion of antibiotic resistance genes identified among taxa in fecal metagenomes. Nearly all ARGs could be assigned to Enterobacteriaceae (red), Enterococcaceae (yellow), or Staphylococcaceae (green), though some could not be confidently assigned to one organism (purple). b Infants transferred between hospitals participating in this study demonstrated rapid colonization by bacteria harboring hospital-specific ARGs after their transfer date (circle, age in days at transfer). All four transferred infants were assigned to the feeding protocol including B. infantis EVC001, and the amount of hospital-specific ARGs trended lower and similar to other infants in the unit that received B. infantis EVC001 (teal line, confidence intervals), relative to infants who did not receive the probiotic (grey line, confidence intervals). c Infants at the two hospitals developed similar patterns of ARG colonization, with more than 100 different unique ARGs detected. Infants acquired ARGs throughout their stay, though infants fed B. infantis EVC001 tended to acquire fewer unique ARGs (teal), relative to infants who did not receive the probiotic (grey). d Samples from infants fed 
B. infantis EVC001 also exhibited a lower overall relative abundance of ARGs (teal), as a proportion of their microbiome, compared to samples from infants who did not receive the probiotic (grey). (P values indicated by asterisks, $* * \star *, P<0.0001$ )

Figure 6

A

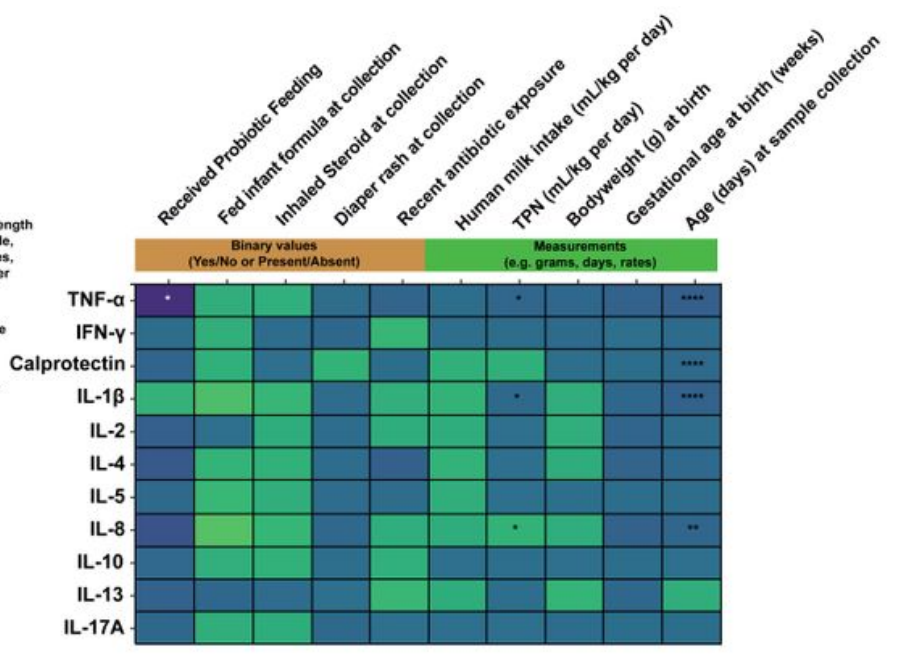

B
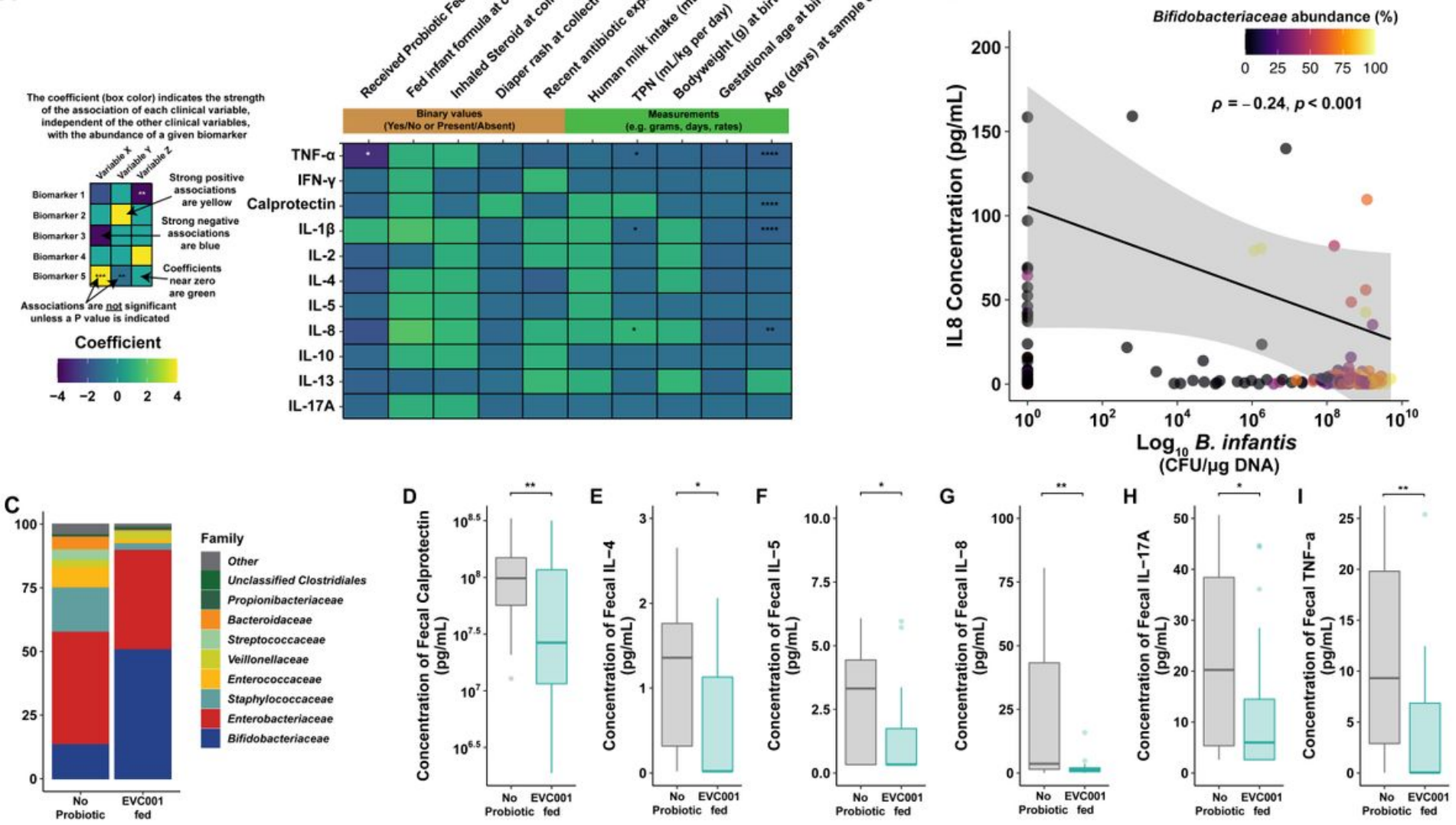

\section{Figure 6}

Proinflammatory biomarkers are significantly lower in preterm infants fed B. infantis EVC001. a Multivariate analysis by linear models (MaAsLin2) was used to assess the effect that each clinical variable had, independently, on proinflammatory biomarker concentrations. Probiotic feeding (B. infantis EVC001) was significantly associated with lower TNFa concentrations $(-1.06 \mathrm{pg} / \mathrm{mL}$ per $\mathrm{kg}$ per day; FDRcorrected P value $=0.0011)$. TPN $(\mathrm{mL} / \mathrm{kg}$ * day) correlated with increased IL-8 production $(1.25 \mathrm{pg} / \mathrm{mL}$ per unit increase; FDR-corrected $P$ value $=0.02)$ and a decrease of IL-1 $\beta$ and TNFa $(0.064$ and $0.1 \mathrm{pg} / \mathrm{mL}$ * day; FDR-corrected $P$ value $=0.02,0.02$, respectively). Day of life at sampling associated with decreased calprotectin, IL-1 $\beta, \mathrm{IL}-8$, and TNFa production at a rate of $0.04,0.07,0.1$, and $0.08 \mathrm{pg} / \mathrm{mL}$ * day, respectively (FDR-corrected $\mathrm{P}$ value $=0.0001,0.0001,0.002$, and 0.0001 , respectively). No significant associations between inhaled steroid exposure, diaper rash and treatment, antibiotic exposure, formula feeding, human milk consumption, birth weight, or gestational age at birth was identified. b Significantly decreased proinflammatory cytokine, IL-8 correlated with B. infantis EVC001 absolute abundance (P = $0.001, \rho=-0.24)$. $c$ The mean relative abundance of bacterial families in samples analyzed at the $\sim 34$ week timepoint, colored by family, is shown. Mean and standard deviations are available in Table S5. d-i Box plots represent fecal calprotectin and proinflammatory cytokine concentrations $[\mathrm{pg} / \mathrm{mg}]$ from infants 
not fed B. infantis EVCO01 $(n=28)$ and EVC001-fed infants $(n=29)$ at 34 weeks corrected gestational age. Calprotectin and cytokine concentrations were measured in duplicate using MesoScale Discoveries $\mathrm{R}$ and U-plex, respectively. Statistical analysis was completed using Wilcoxon rank sum test. P-values were adjusted using the FDR method and considered to be statistically significantly decreased if $\star \star \star \star ~ P<$ $0.0001 ; * * * \mathrm{P}<0.001 ; * * \mathrm{P}<0.01 ; \mathrm{P}<0.05$.
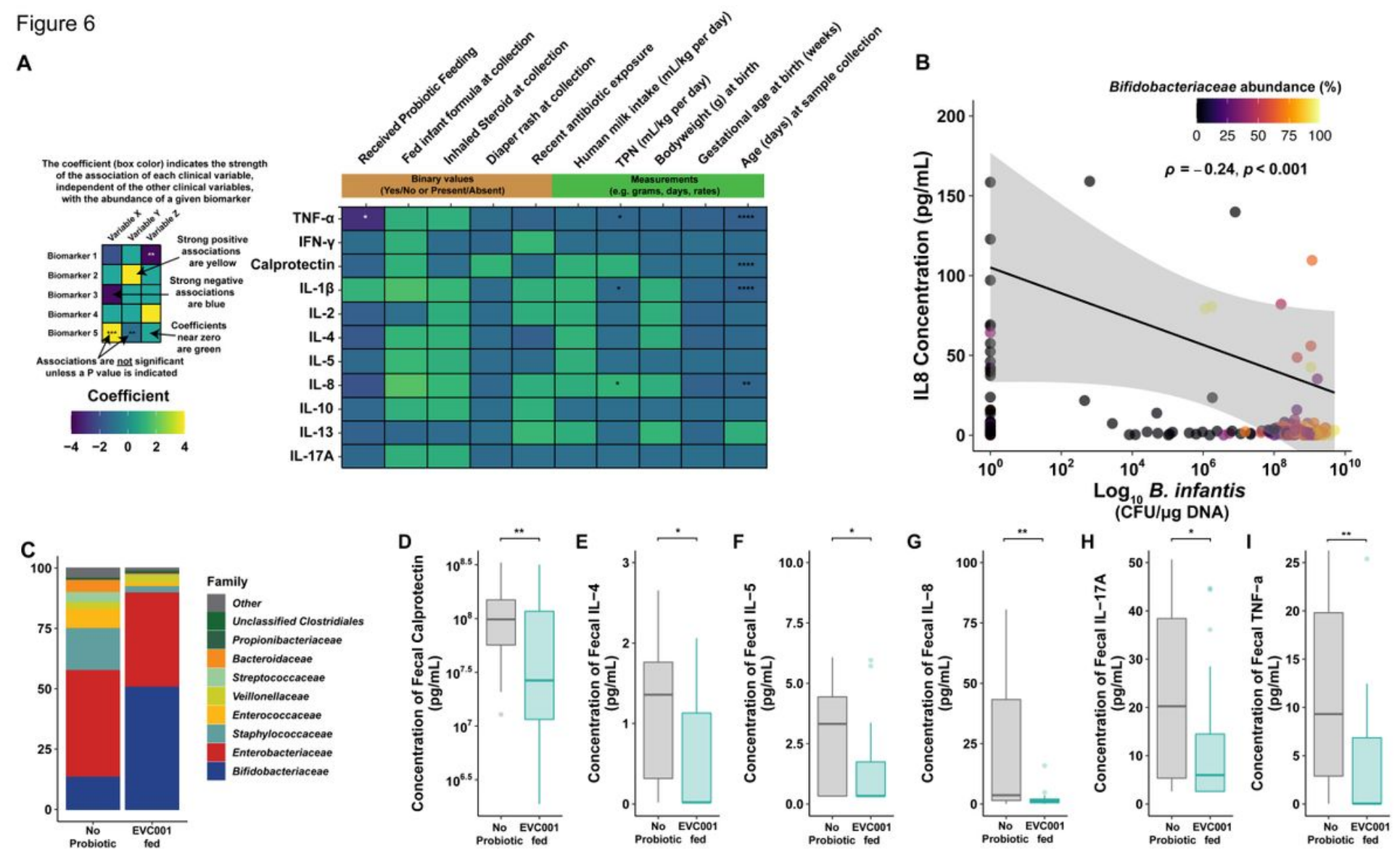

\section{Figure 6}

Proinflammatory biomarkers are significantly lower in preterm infants fed B. infantis EVC001. a Multivariate analysis by linear models (MaAsLin2) was used to assess the effect that each clinical variable had, independently, on proinflammatory biomarker concentrations. Probiotic feeding (B. infantis EVC001) was significantly associated with lower TNFa concentrations $(-1.06 \mathrm{pg} / \mathrm{mL}$ per kg per day; FDRcorrected $\mathrm{P}$ value $=0.0011)$. TPN $(\mathrm{mL} / \mathrm{kg}$ * day) correlated with increased IL-8 production $(1.25 \mathrm{pg} / \mathrm{mL}$ per unit increase; FDR-corrected $P$ value $=0.02)$ and a decrease of IL-1 $\beta$ and TNFa $(0.064$ and $0.1 \mathrm{pg} / \mathrm{mL}$ * day; FDR-corrected $P$ value $=0.02,0.02$, respectively). Day of life at sampling associated with decreased calprotectin, IL-1 $1 \beta, \mathrm{IL}-8$, and TNFa production at a rate of $0.04,0.07,0.1$, and $0.08 \mathrm{pg} / \mathrm{mL}$ * day, respectively (FDR-corrected $\mathrm{P}$ value $=0.0001,0.0001,0.002$, and 0.0001 , respectively). No significant associations between inhaled steroid exposure, diaper rash and treatment, antibiotic exposure, formula feeding, human milk consumption, birth weight, or gestational age at birth was identified. b Significantly decreased proinflammatory cytokine, IL-8 correlated with B. infantis EVC001 absolute abundance ( $\mathrm{P}=$ $0.001, \rho=-0.24)$. $c$ The mean relative abundance of bacterial families in samples analyzed at the $\sim 34$ 
week timepoint, colored by family, is shown. Mean and standard deviations are available in Table S5. d-i Box plots represent fecal calprotectin and proinflammatory cytokine concentrations [pg/mg] from infants not fed B. infantis EVC001 ( $n=28)$ and EVC001-fed infants $(n=29)$ at 34 weeks corrected gestational age. Calprotectin and cytokine concentrations were measured in duplicate using MesoScale Discoveries $\mathrm{R}$ and U-plex, respectively. Statistical analysis was completed using Wilcoxon rank sum test. P-values were adjusted using the FDR method and considered to be statistically significantly decreased if $\star \star \star \star ~ P<$ $0.0001 ; * \star \star P<0.001 ; * \star P<0.01 ; P<0.05$.

\section{Supplementary Files}

This is a list of supplementary files associated with this preprint. Click to download.

- SupplementaryTables.pdf

- SupplementaryTables.pdf

- SupplementaryFiguresandLegends.pdf

- SupplementaryFiguresandLegends.pdf 